\title{
ON THE BOREL COHOMOLOGY OF FREE LOOP SPACES
}

\author{
IVER OTTOSEN*
}

\begin{abstract}
Let $X$ be a space and let $K=H^{*}\left(X ; \mathrm{F}_{p}\right)$ where $p$ is an odd prime. We construct functors $\bar{\Omega}$ and $\ell$ which approximate cohomology of the free loop space $\Lambda X$ as follows: There are homomorphisms $\bar{\Omega}(K) \rightarrow H^{*}\left(\Lambda X ; \mathrm{F}_{p}\right)$ and $\ell(K) \rightarrow H^{*}\left(E \mathrm{~T} \times_{\mathrm{T}} \Lambda X ; \mathrm{F}_{p}\right)$. These are isomorphisms when $X$ is a product of Eilenberg-MacLane spaces of type $K\left(\mathrm{~F}_{p}, n\right)$ for $n \geq 1$.
\end{abstract}

\section{Introduction}

Let $X$ be a topological space and $R$ a ring. The circle group T acts on the free loop space $\Lambda X$ by rotation of loops. The associated Borel cohomology groups are called string cohomology of $X$ [4]. We denote them as follows:

$$
H_{s t}^{*}(X ; R)=H^{*}\left(E \mathrm{~T} \times_{\mathrm{T}} \Lambda X ; R\right) .
$$

String cohomology as well as non equivariant cohomology of free loop spaces play a central role in geometry and topology. It is however often not possible to compute such cohomology groups.

When $R=\mathrm{F}_{2}=\mathrm{Z} / 2$, M. Bökstedt and I found functors of $H^{*}(X)$ which approximate $H_{s t}^{*}(X)$ and $H^{*}(\Lambda X)$ [2]. The purpose of this paper is to generalize these functors to the case $R=\mathrm{F}_{p}=\mathrm{Z} / p$ where $p$ is any of the odd primes. Certain algebra generators in string cohomology are more difficult to construct in the odd primary case. Hence method and strategy differs from [2] at various places.

The following application of the functors $\bar{\Omega}$ and $\ell$ will appear in the near future. There are two Bousfield cohomology spectral sequences. One converging to $H^{*}(\Lambda X)$ and the other converging to $H_{s t}^{*}(X)$. The $E_{2}$ term of the first is isomorphic to the (non Abelian) derived functors of $\bar{\Omega}$ and the $E_{2}$ term of the second is isomorphic to the derived functors of $\ell$.

\footnotetext{
* The author was supported by the European Union TMR network ERB FMRX CT-97-0107: Algebraic K-theory, Linear Algebraic Groups and Related Structures.

Received January 15, 2002.
} 
Notation. Fix an odd prime $p$. We use $\mathrm{F}_{p}$-coefficients everywhere unless otherwise is specified. $\mathscr{A}$ denotes the mod $p$ Steenrod algebra, $\mathscr{U}$ the category of unstable $\mathscr{A}$-modules and $\mathscr{K}$ the category of unstable $\mathscr{A}$-algebras. We let $\mathscr{A l g}$ denote the following category. An object in $\mathscr{A} l g$ is a non-negatively graded $\mathrm{F}_{p}$-algebra $A$ with the property that if $a \in A$ and $|a|=0$ then $a=a^{p}$. The category of differential graded $\mathrm{F}_{p}$-algebras is denoted $D G A$. For any $A \in \mathscr{A l} l g$ we define $\sigma: A \rightarrow \mathrm{F}_{p}$ by $\sigma(x)=1$ for $|x|$ odd and $\sigma(x)=0$ for $|x|$ even. We also define $\hat{\sigma}: A \rightarrow \mathrm{F}_{p}$ by $\hat{\sigma}(x)=1-\sigma(x)$. The circle group is denoted $\mathrm{T}$.

\section{The approximation functor $\bar{\Omega}$}

In this section we define a functor $\bar{\Omega}: \mathscr{F} \rightarrow \mathscr{A} l g$ which approximates the cohomology ring $H^{*}(\Lambda X)$ when applied to $H^{*} X$. Here $\mathscr{F}$ is a certain category which lies between $\mathscr{K}$ and $\mathscr{A} \lg$. The functor $\bar{\Omega}$ lifts to an endofunctor on $\mathscr{K}$ which is nothing but an explicit description of Lannes' division functor $\left(-: H^{*}(\mathrm{~T})\right)_{\mathscr{C}}$ introduced in [5].

Definition 2.1. Let $\mathscr{F}$ denote the following category. An object in $\mathscr{F}$ is an object $A \in \mathscr{A} l g$ which is equipped with an $\mathrm{F}_{p}$-linear map $\lambda: A \rightarrow A$ with the following properties:

- $|\lambda x|=p(|x|-1)+1$ for all $x \in A$.

- $\lambda x=x$ when $|x|=1$ and $\lambda x=0$ when $|x|$ is even.

- $\lambda(x y)=\lambda(x) y^{p}+x^{p} \lambda(y)$ for all $x, y \in A$.

Furthermore $A$ is equipped with an $\mathrm{F}_{p}$-linear map $\beta: A \rightarrow A$ of degree 1 with the following properties:

- $\beta \circ \beta=0$.

- $\beta(x y)=\beta(x) y+(-1)^{|x|} x \beta(y)$ for all $x, y \in A$.

A morphism $f: A \rightarrow A^{\prime}$ in $\mathscr{F}$ is a morphism in $\mathscr{A} \lg$ such that $f(\lambda x)=$ $\lambda^{\prime} f(x)$ and $f(\beta x)=\beta^{\prime} f(x)$.

Remark 2.2. There are forgetful functors $\mathscr{K} \rightarrow \mathscr{F}$ and $\mathscr{F} \rightarrow \mathscr{A} l g$. For an object $K$ in $\mathscr{K}$ the map $\lambda: K \rightarrow K$ is defined by $\lambda x=P^{(|x|-1) / 2} x$ when $|x|$ is odd. The map $\beta$ is the Bockstein operation.

We let $\Lambda(v)$ denote the object $H^{*}(\mathrm{~T})$ in $\mathscr{K}$. There is an associative and commutative coproduct $\delta: \Lambda(v) \rightarrow \Lambda(v) \otimes \Lambda(v) ; v \mapsto 1 \otimes v+v \otimes 1$. It comes from the product on $\mathrm{T}$ and has counit $\gamma: \Lambda(v) \rightarrow \mathrm{F}_{p}$ coming from the unit $1 \rightarrow \mathrm{T}$.

Let $\perp: \mathscr{K} \rightarrow \mathscr{K}$ be the functor given by $A \mapsto \Lambda(v) \otimes A$. The coproduct and counit above define natural transformations $\delta: \perp \rightarrow \perp^{2}$ and $\gamma: \perp \rightarrow I d$ 
such that $(\perp, \delta, \gamma)$ is a comonad. $\mathrm{A} \perp$-coalgebra is an object $K$ in $\mathscr{K}$ equipped with a morphism $f: K \rightarrow \perp(K)$ such that the following diagrams commute:

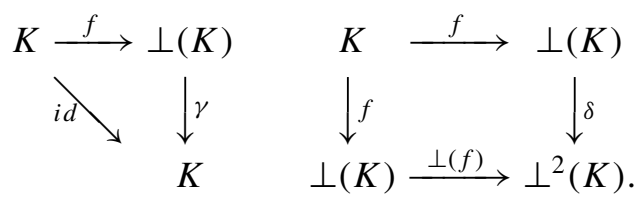

Examples of $\perp$-coalgebras are cohomology of T-spaces.

Proposition 2.3. If $K$ is a $\perp$-coalgebra with structure map $f: K \rightarrow \perp(K)$ then $K$ is a graded commutative DGA with degree -1 differential d given by

$$
f(x)=1 \otimes x+v \otimes d x, \quad x \in K .
$$

Furthermore, $d\left(P^{i} x\right)=P^{i} d x$ for each $i \geq 0$ and $d(\beta x)=-\beta d(x)$. In particular $d(\lambda x)=(d x)^{p}$ and $d(\beta \lambda x)=0$.

Proof. By the left of the above diagrams $f$ may be expanded as stated. By the right diagram $d \circ d=0$. Since $f$ is a morphism in $\mathscr{K}$ we see that $d$ is $\mathrm{F}_{p}$-linear, a derivation over the identity and that the stated relations hold.

Proposition 2.4. Assume that the functor $\perp: \mathscr{K} \rightarrow \mathscr{K}$ has a left adjoint $\top: \mathscr{K} \rightarrow \mathscr{K}$. Then there is a natural $\perp$-coalgebra structure $\eta: \top \rightarrow \perp \top$ on $\top$. For an object $B \in \mathscr{K}$ the map $\eta_{B}$ is the image of the identity under the composite

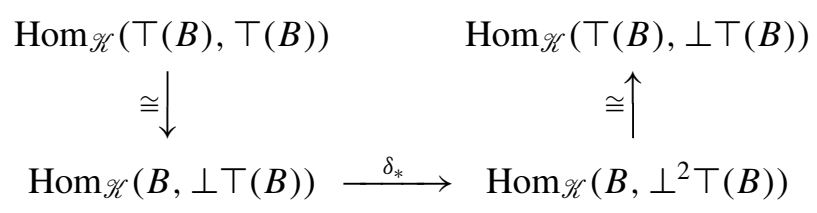

Proof. This is formally the same as the proof of [11] Proposition 3.4.

Definition 2.5. For $A \in \mathscr{F}$ we define $\bar{\Omega}(A)$ as the quotient of the free graded commutative and unital $A$-algebra on generators

$$
d x \quad \text { for } \quad x \in A
$$

where $|d x|=|x|-1$, by the ideal generated by the elements

$$
\begin{aligned}
& d(x+y)-d x-d y, \\
& d(x y)-d(x) y-(-1)^{|x|} x d(y), \\
& d(\lambda x)-(d x)^{p}, \\
& d(\beta \lambda x) .
\end{aligned}
$$


Note that $\bar{\Omega}(A)$ is non-negatively graded since $d\left(x^{p}\right)=0$. We have defined a functor $\bar{\Omega}: \mathscr{F} \rightarrow \mathscr{A l g}$.

Proposition 2.6. The functor $\bar{\Omega}: \mathscr{F} \rightarrow \mathscr{A l g}$ lifts to a functor $\bar{\Omega}:$ $\mathscr{K} \rightarrow \mathscr{K}$. Explicitely the $\mathscr{A}$-action on $\bar{\Omega}(K)$ is given by $\theta(x)=\theta x$ and $\theta(d x)=(-1)^{|\theta|} d(\theta x)$ for $x \in K$ and $\theta \in \mathscr{A}$ and the Cartan formula. The differential d on $\bar{\Omega}(K)$ is graded $\mathscr{A}$-linear.

Proof. Let $d K$ denote the graded $\mathrm{F}_{p}$-vector space given by $(d K)^{n}=K^{n+1}$. We write $d x$ for the element in $d K$ corresponding to $x$ in $K$ hence $d(x+y)=$ $d x+d y$. We define an $\mathscr{A}$-module structure on $d K$ by $P^{i} d x=d P^{i} x$ and $\beta d x=-d \beta x$. Let $S(d K)$ denote the free graded commutative algebra on the $\mathrm{F}_{p}$-vector space $d K$. By the Cartan formula $S(d K)$ is an $\mathscr{A}$-algebra and the symmetric product $K \odot S(d K)$ is an $\mathscr{A}$-algebra. By definition $\bar{\Omega}(K)=$ $K \odot S(d K) / I$ where $I$ is the ideal generated by

$$
\begin{aligned}
& 1 \odot d(x y)-d(x) \odot y-(-1)^{|x|} x \odot d(y), \\
& 1 \odot\left(d(\lambda x)-(d x)^{p}\right), \\
& 1 \odot d(\beta \lambda x) .
\end{aligned}
$$

We verify that $\mathscr{A} \cdot I \subseteq I$ such that $\bar{\Omega}(K)$ is an $\mathscr{A}$-algebra. We have

$$
\begin{aligned}
& P^{n}\left(1 \odot d(x y)-d x \odot y-(-1)^{|x|} x \odot d y\right) \\
& \quad=\sum_{i+j=n}\left(1 \odot d\left(P^{i}(x) P^{j}(y)\right)-d P^{i} x \odot P^{j} y-(-1)^{|x|} P^{i} x \odot d P^{j} y\right)
\end{aligned}
$$

which is in $I$ by (5) since the degree of $P^{i}$ is even. Further

$$
\begin{aligned}
& \beta\left(1 \odot d(x y)-d x \odot y-(-1)^{|x|} x \odot d y\right) \\
& =-\left(1 \odot d(\beta(x) y)-d \beta x \odot y-(-1)^{|\beta x|} \beta x \odot d y\right) \\
& \quad-(-1)^{|x|}\left(1 \odot d(x \beta y)-d x \odot \beta y-(-1)^{|x|} x \odot d \beta y\right)
\end{aligned}
$$

which is also in $I$ by (5).

In any $\mathscr{A}$-algebra one has $P^{i}\left(a^{p}\right)=\left(P^{i / p} a\right)^{p}$ when $i=0 \bmod p$ and zero otherwise, since this is a consequence of the Cartan formula alone. So by Lemma 2.7 we have the following relation in $S(d K)$ when $i=0 \bmod p$ :

$$
P^{i}\left(d(\lambda x)-(d x)^{p}\right)=d\left(P^{i} \lambda x\right)-\left(P^{i / p} d x\right)^{p}=d\left(\lambda P^{i / p} x\right)-\left(d P^{i / p} x\right)^{p} .
$$

For $i \neq 0 \bmod p$ we get zero. So $P^{i}$ applied to an element of the form (6) lies in $I$. If we apply $\beta$ to such an element we also land in $I$ by (7). Finally Lemma 2.7 shows that $P^{i}(1 \odot d(\beta \lambda x)) \in I$ and trivially $\beta(1 \odot d(\beta \lambda x)) \in I$. 
We verify that $\bar{\Omega}(K) \in \mathcal{U}$. We must show that $P^{i} d x=0$ if $2 i>|x|-1$. This holds if $2 i>|x|$ since $K \in \mathcal{U}$. If $2 i=|x|$ we have $P^{i} d x=d P^{i} x=$ $d\left(x^{p}\right)=0$. We must also show that $\beta P^{i} d x=0$ when $2 i+1>|x|-1$. This holds if $2 i+1>|x|$ since $K \in \mathcal{U}$ and if $2 i+1=|x|$ we have $\beta P^{i} d x=-d \beta P^{i} x=-d \beta \lambda x=0$. Since the action on products are by the Cartan formula we have shown that $\bar{\Omega}(K) \in \mathcal{U}$.

Finally we check that $\bar{\Omega}(K) \in \mathscr{K}$. The Cartan formula holds by definition. For $|x|$ odd we have $P^{|d x| / 2}(d x)=d \lambda x=(d x)^{p}$ and the result follows.

Lemma 2.7. For any unstable $\mathscr{A}$-algebra $K$ and $x \in K$ the following equations hold.

$$
\begin{gathered}
P^{i} \lambda x= \begin{cases}\lambda\left(P^{\frac{i}{p}} x\right), & i=0 \bmod p \\
0, & \text { otherwise }\end{cases} \\
P^{i} \beta \lambda x= \begin{cases}\beta \lambda\left(P^{\frac{i}{p}} x\right), & i=0 \bmod p \\
\left(\beta P^{\frac{i-1}{p}} x\right)^{p}, & i=1 \bmod p \\
0, & \text { otherwise }\end{cases}
\end{gathered}
$$

Proof. We just prove (8) since the proof of (9) is similar. When $|x|$ is even both sides in the equation are zero. Assume that $|x|$ is odd. By the instability condition $P^{i} \lambda x=0$ when $2 i>p(|x|-1)+1$. When $i$ is divisible by $p$ this inequality implies $2 i \geq p(|x|-1)+p$ or $\frac{2 i}{p} \geq|x|$ and since $|x|$ is odd $\frac{2 i}{p}>|x|$. So $P^{i / p} x=0$ and the equation holds in this case. If $2 i=p(|x|-1)$ then $P^{i} \lambda x=\lambda^{2} x=\lambda\left(P^{i / p} x\right)$.

Finally assume that $2 i<p(|x|-1)$. Then we can apply the Adem relation:

$$
P^{i} P^{\frac{|x|-1}{2}} x=\sum_{t=0}^{\left[\frac{i}{p}\right]}(-1)^{i+t}\left(\begin{array}{c}
(p-1)\left(\frac{|x|-1}{2}-t\right)-1 \\
i-p t
\end{array}\right) P^{i+\frac{|x|-1}{2}-t} P^{t} x .
$$

The instability condition shows that $P^{i+\frac{|x|-1}{2}-t} P^{t} x=0$ unless $i \leq p t$. But the binomial coefficient is zero when $i<p t$. So we get zero when $i \neq 0 \bmod p$ and the term corresponding to $t=i / p$ when $i=0 \bmod p$.

Proposition 2.8. The functor $\bar{\Omega}: \mathscr{K} \rightarrow \mathscr{K}$ is left adjoint to $\perp: \mathscr{K} \rightarrow \mathscr{K}$; $B \mapsto H^{*}(\mathrm{~T}) \otimes B$. Thus there is an equivalence offunctors $\bar{\Omega} \cong\left(-: H^{*}(\mathrm{~T})\right)_{\mathscr{K}}$. The differential $d: \bar{\Omega}(A) \rightarrow \bar{\Omega}(A)$, associated to the natural $\perp$-coalgebra structure, is given by $d(x)=d x$ for $x \in A$. 
Proof. We can define natural maps as follows where $x \in A$ :

$$
\begin{gathered}
F: \operatorname{Hom}_{\mathscr{K}}(\bar{\Omega}(A), B) \rightleftarrows \operatorname{Hom}_{K}(A, \perp(B)): G \\
F(f)(x)=1 \otimes f(x)+v \otimes f(d x), \\
G(g)(x)=\gamma \circ g(x), \quad G(g)(d x)=(\alpha \otimes 1) \circ g(x)
\end{gathered}
$$

where $\alpha: \Lambda(v) \rightarrow \mathrm{F}_{p}$ is the additive map of degree -1 given by $v \mapsto 1$ and $1 \mapsto 0$. It is easy to verify that $F \circ G=i d$ and $G \circ F=i d$. The description of $d$ follows by using these explicit adjunction formulas in the composite defining $\eta$ in Proposition 2.4.

Proposition 2.9. For any space $X$ there is a morphism in $\mathscr{K}$ (and in $D G A$ )

$$
e: \bar{\Omega}\left(H^{*} X\right) \rightarrow H^{*}(\Lambda X) ; \quad e(x)=e v_{0}^{*}(x) ; \quad e(d x)=d e v_{0}^{*}(x)
$$

where e $v_{0}: \Lambda X \rightarrow X ; \omega \mapsto \omega(1)$. This morphism is natural in $X$ and it is an isomorphism if $X=K\left(\mathrm{~F}_{p}, n\right)$ with $n \geq 0$. If $H_{*} X$ is of finite type and $Y$ is any space then there is a commutative diagram

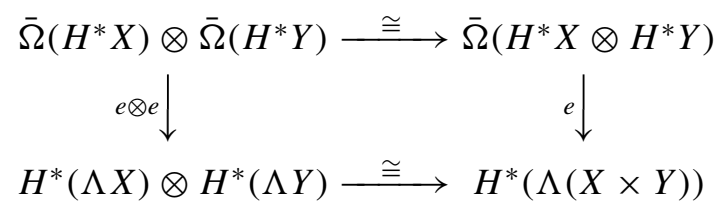

where the lower horizontal map is the Künneth isomorphism.

Proof. The proof of Proposition 3.9 in [11] goes through with the obvious changes. Thus the isomorphism statement is a consequence of [5] 1.11.

\section{The approximation functor $\ell$}

In this section we describe the functor $\ell: \mathscr{F} \rightarrow \mathscr{A l g}$ which gives an approximation to $H^{*}\left(E \mathrm{~T} \times_{\mathrm{T}} \Lambda X\right)$ when applied to $H^{*} X$. We also define a natural transformation $Q: \ell \rightarrow \bar{\Omega}$ which corresponds to the map $H^{*}\left(E \mathrm{~T} \times_{\mathrm{T}} \Lambda X\right) \rightarrow$ $H^{*}(\Lambda X)$ induced by the quotient. We do however not go into the topological interpretations here.

Definition 3.1. For $A \in \mathscr{F}$ we define $\ell(A)$ as the free graded commutative $\mathrm{F}_{p}$-algebra on generators $\phi(x), q(x), \delta(x)$ for $x \in A$ and $u$ of degrees

$$
\begin{array}{lll}
|\phi(x)|=p|x|-\sigma(x)(p-1), & & |q(x)|=p|x|-1-\sigma(x)(p-3), \\
|\delta(x)|=|x|-1, & & |u|=2
\end{array}
$$


modulo the ideal generated by

$$
\phi(x+y)-\phi(x)-\phi(y)+\sigma(x) \sum_{i=0}^{p-2}(-1)^{i} \delta(x)^{i} \delta(y)^{p-2-i} \delta(x y),
$$

(11) $\delta(x+y)-\delta(x)-\delta(y)$

$$
q(x+y)-q(x)-q(y)+\hat{\sigma}(x) \sum_{i=1}^{p-1}(-1)^{i} \frac{1}{i} \delta\left(x^{i} y^{p-i}\right),
$$

(14) $\phi(a b)-\left(-u^{p-1}\right)^{\sigma(a) \sigma(b)} \phi(a) \phi(b)$,

$$
\begin{aligned}
(-1)^{\sigma(a) \hat{\sigma}(c)} \delta(a) \delta(b c)+(-1)^{\sigma(b) \hat{\sigma}(a)} \delta(b) \delta(c a) \\
+(-1)^{\sigma(c) \hat{\sigma}(b)} \delta(c) \delta(a b),
\end{aligned}
$$

(19) $\delta(x) u$,

(20) $q(\beta \lambda x)$

(21) $\quad \delta\left(x^{p}\right)$

where $a, b, c, x, y \in K$ and $|x|=|y|$.

REMARK 3.2. We have some immediate consequences of these relations: By (10), (11) and (20) we have $\phi(0)=q(0)=\delta(0)=0$. By (14) and (15) we have $q\left(a^{n}\right)=n \phi(a)^{n-1} q(a)$ such that $q\left(a^{p}\right)=0$. By (21) we have $\delta(1)=0$ so by (21) and (17) we find $\delta(\lambda b)=\delta(b)^{p}$. By (18) and $\delta(1)=0$ we have $\delta(\beta \lambda b)=0$. By (14), (15) and (17) the algebra $\ell(A)$ is unital with unit $\phi(1)$.

Since $\delta\left(x^{p}\right)=q\left(x^{p}\right)=0$ we see that $\ell(A)$ is non-negatively graded. We have defined a functor $\ell: \mathscr{F} \rightarrow \mathscr{A l g}$.

LemmA 3.3. Let $K \in \mathscr{F}$ and $x, y \in K$ with $|x|=|y|=n$. The following relations hold in $\bar{\Omega}(K)$ :

$$
\begin{aligned}
& \sum_{i=1}^{p-1}(-1)^{i+1} \frac{1}{i} d\left(x^{i} y^{p-i}\right) \\
& \quad=(x+y)^{p-1} d(x+y)-x^{p-1} d x-y^{p-1} d y, \quad n \text { even }
\end{aligned}
$$




$$
\begin{aligned}
& \sum_{j=0}^{p-2}(-1)^{j+1}(d x)^{j}(d y)^{p-2-j} d(x y) \\
& \quad=(d(x+y))^{p-1}(x+y)-(d x)^{p-1} x-(d y)^{p-1} y, \quad \text { nodd }
\end{aligned}
$$

Proof. We verify (22) and omit the proof of (23) which is similar. Since $d$ is a derivation we have

$$
\sum_{i=1}^{p-1}(-1)^{i+1} \frac{1}{i} d\left(x^{i} y^{p-i}\right)=\sum_{i=1}^{p-1}(-1)^{i+1}\left(x^{i-1} y^{p-1} d x-x^{i} y^{p-i-1} d y\right) .
$$

By splitting the sum in two at the minus sign and substituting $j=i-1$ in the first of the resulting sums we see that the above equals the following:

$$
\begin{aligned}
\sum_{j=0}^{p-2}(-1)^{j} x^{j} y^{p-j-1} d x+\sum_{i=1}^{p-1}(-1)^{i} x^{i} y^{p-i-1} d y \\
=\sum_{t=0}^{p-1}(-1)^{t} x^{t} y^{p-t-1}(d x+d y)-x^{p-1} d x-y^{p-1} d y .
\end{aligned}
$$

For $0 \leq t \leq p-1$ we have that $t$ ! is invertible in $\mathrm{F}_{p}$ and also

$$
\left(\begin{array}{c}
p-1 \\
t
\end{array}\right) t !=(p-1)(p-2) \ldots(p-t)=(-1)^{t} t ! \bmod p .
$$

Thus we have $\left(\begin{array}{c}p-1 \\ t\end{array}\right)=(-1)^{t}$. Substituting this in the above and using the binomial formula the result follows.

Proposition 3.4. For $A \in \mathscr{F}$ there is a natural morphism in $\mathscr{A l g}$ as follows:

$$
\begin{aligned}
Q: \ell(A) \rightarrow \bar{\Omega}(A) ; \quad \phi(x) & \mapsto x^{p}+\lambda x-x(d x)^{p-1}, \\
\delta(x) & \mapsto d x, \quad q(x) \mapsto x^{p-1} d x+\beta \lambda x, \quad u \mapsto 0 .
\end{aligned}
$$

Furthermore, $\operatorname{Im}(Q) \subseteq \operatorname{ker}(d: \bar{\Omega}(A) \rightarrow \bar{\Omega}(A))$.

Proof. We check that the formulas for $Q$ map the relations (10)-(21) to zero. Formula (23) and the additivity of $x \mapsto x^{p}$ shows that (10) is mapped to zero. It is trivial that (11) is mapped to zero. By (22) and the additivity of $x \mapsto \beta \lambda x$ it follows that (12) is mapped to zero. 
Taking the derivative of products and permuting factors we find the following equations:

$$
\begin{aligned}
& d(a) d(b c)=d(a) d(b) c+(-1)^{\sigma(b)} d(a) b d(c), \\
& d(b) d(c a)=(-1)^{\sigma(a)(\hat{\sigma}(b)+\hat{\sigma}(c))} a d(b) d(c) \\
&+(-1)^{\sigma(c)+\hat{\sigma}(a)(\hat{\sigma}(b)+\sigma(c))} d(a) d(b) c
\end{aligned}
$$

After some reductions (13) follows from these.

One easily checks that (14) and (15) are mapped to zero in each of the cases $\sigma(a)=\sigma(b)=0, \sigma(a)=\sigma(b)=1$ and $\sigma(a)=\hat{\sigma}(b)=1$. It also follows by small direct computations that (16)-(21) are mapped to zero.

\section{The morphism $Q$ and cohomology of $\bar{\Omega}(A)$}

In this section we define an additive transformation $\tau: \bar{\Omega} \rightarrow \ell$ which corresponds to the T-transfer from $H^{*}(\Lambda X)$ to $H^{*}\left(E \mathrm{~T} \times_{\mathrm{T}} \Lambda X\right)$. The map $Q$ gives a morphism from $\ell(A) /(u)$ to the cycles in $\bar{\Omega}(A)$. Via this a map $\Phi$ similar to the Cartier map [3] is defined. It turns out that $\ell(A) /(u) \cong \operatorname{ker}(d)$ when $\Phi$ is an isomorphism. Parts of the material presented here correspond to section 8 in [2]. We let $A$ denote an object in $\mathscr{F}$.

Definition 4.1. Let $I_{\delta}(A) \subseteq \ell(A)$ denote the ideal $I_{\delta}(A)=(\delta(x) \mid x \in A)$.

Proposition 4.2. There is an $\mathrm{F}_{p}$-linear map of degree -1 as follows

$$
\tau: \bar{\Omega}(A) \rightarrow \ell(A) ; \quad a_{0} d a_{1} \ldots d a_{n} \mapsto \delta\left(a_{0}\right) \delta\left(a_{1}\right) \ldots \delta\left(a_{n}\right), \quad a_{0} \mapsto \delta\left(a_{0}\right)
$$

where $a_{i} \in A$ for each $i$. It has the following properties:

$\tau(Q(\alpha) \beta)=(-1)^{|\alpha|} \alpha \tau(\beta)$ for $\alpha \in \ell(A), \beta \in \bar{\Omega}(A), \quad Q \circ \tau=d, \quad \tau \circ Q=0$.

Note that $\tau \circ d=0$ and $\operatorname{Im}(\tau)=I_{\delta}(A)$.

Proof. We must show that $\tau$ is well defined. The relations arising from (1), (3) and (4) are respected since we have the same relations in $\ell(K)$ with $d$ replaced by $\delta$. We must verify that the following relation is respected:

$$
\begin{aligned}
& a_{0} d a_{1} \ldots d a_{i-1} d\left(a_{i} a_{i+1}\right) d a_{i+2} \ldots d a_{n} \\
& \quad=(-1)^{\left(k+\hat{\sigma}\left(a_{i}\right)\right) \sigma\left(a_{i+1}\right)} a_{0} a_{i+1} d a_{1} \ldots d a_{i} d a_{i+2} \ldots d a_{n} \\
& \quad+(-1)^{(k+1) \sigma\left(a_{i}\right)} a_{0} a_{i} d a_{1} \ldots d a_{i-1} d a_{i+1} \ldots d a_{n}
\end{aligned}
$$


where $k=\left|d a_{1} \ldots d a_{i-1}\right|$. It suffices to check that

$$
x d(y z)=(-1)^{\hat{\sigma}(y) \sigma(z)} x z d(y)+(-1)^{\sigma(y)} x y d(z)
$$

is respected. This follows by (13) after some work with the signs.

By definition we have $Q \circ \tau=0$. By direct computations one sees that $\tau(Q(\alpha) \beta)=(-1)^{|\alpha|} \alpha \tau(\beta)$ when $\alpha$ equals $\phi(x), q(x)$ or $\delta(x)$ and $\beta$ equals $a_{0} d a_{1} \ldots d a_{n}$ or $a_{0}$. The general case follows from this. In particular $\tau \circ Q=0$ since $\tau(1)=0$.

Definition 4.3. Let $\mathscr{L}(A)=\ell(A) /(u)$ and $\widetilde{\Omega}(A)=\mathscr{L}(A) / I_{\delta}(A)$. Explicitely, $\widetilde{\Omega}(A)$ is the free graded commutative $\mathrm{F}_{p}$-algebra on generators $\phi(x)$, $q(x)$ for $x \in A$ of degrees $|\phi(x)|=p|x|-\sigma(x)(p-1),|q(x)|=p|x|-1-$ $\sigma(p-3)$ modulo the relations that $\phi$ and $q$ are additive and

$$
\begin{aligned}
\phi(a b) & =(1-\sigma(a) \sigma(b)) \phi(a) \phi(b), \\
q(a b) & =\hat{\sigma}(b) q(a) \phi(b)+\hat{\sigma}(a) \phi(a) q(b), \\
\phi(\beta \lambda x) & =q(x)^{p}, \\
q(\beta \lambda x) & =0 .
\end{aligned}
$$

Since $Q\left(I_{\delta}(A)\right) \subseteq d \bar{\Omega}(A)$ we may define an $\mathrm{F}_{p}$-algebra map $\Phi$ by the following diagram where $P$ denotes the canonical projection:

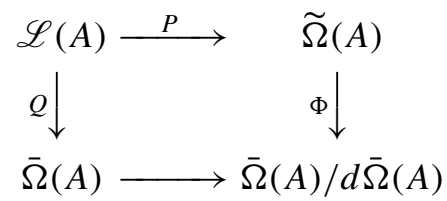

Since $d \circ Q=0$ we have in fact defined a morphism $\Phi: \widetilde{\Omega}(A) \rightarrow H^{*}(\bar{\Omega}(A))$.

Remark 4.4. Since $\tau \circ d=0$ we can define $\tau$ as a map on $\bar{\Omega}(A) / d \bar{\Omega}(A)$. We have a commutative diagram as follows:

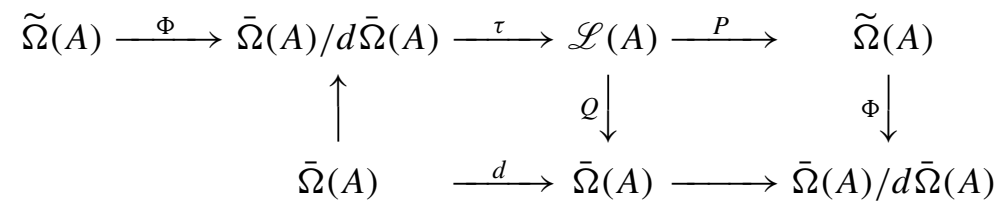

where the composite $\tau \circ \Phi$ vanishes and $\operatorname{ker}(P)=\operatorname{Im}(\tau)$.

THEOREM 4.5. Assume that the map $\Phi: \widetilde{\Omega}(A) \rightarrow H^{*}(\bar{\Omega}(A))$ is an isomorphism. Then so is $Q: \mathscr{L}(A) \rightarrow \operatorname{ker}(d: \bar{\Omega}(A) \rightarrow \bar{\Omega}(A))$. 
Proof. The diagram is formally the same as the one above Theorem 8.5 of [2]. So the same diagram chase gives the result.

There is a filtration $\ell(A) \supseteq u \ell(A) \supseteq u^{2} \ell(A) \supseteq \ldots$ with associated graded object $G r_{*} \ell(A)$ given by $G r_{i} \ell(A)=u^{i} \ell(A) / u^{i+1} \ell(A)$. Consider the following composite of surjective maps:

$$
\ell(A) \stackrel{u^{i} \cdot}{\longrightarrow} u^{i} \ell(A) \longrightarrow G r_{i} \ell(A), \quad i \geq 1
$$

The ideal $I_{\delta}(A)+u \ell(A) \subseteq \ell(A)$ is send to zero so we get a surjective $\mathrm{F}_{p}$-linear $\operatorname{map} u^{i} \cdot: \widetilde{\Omega}(A) \rightarrow G r_{i} \ell(A)$.

Proposition 4.6. For each $i \geq 1$ there is a unique $\mathrm{F}_{p}$-linear map $\Phi_{i}$ such that the following diagram commutes:

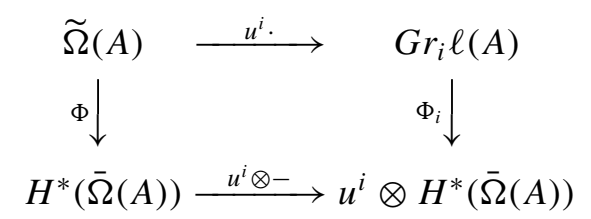

If $\Phi: \widetilde{\Omega}(A) \rightarrow H^{*}(\bar{\Omega}(A))$ is an isomorphism then

$$
G r_{*} \ell(A) \cong \operatorname{ker}(d) \oplus(u \otimes \widetilde{\Omega}(A)) \oplus\left(u^{2} \otimes \widetilde{\Omega}(A)\right) \oplus \cdots .
$$

Proof. The following elements generate the $\mathrm{F}_{p}$-vector space $G r_{i} \ell(A)$ :

$$
u^{i} \phi\left(x_{1}\right) \ldots \phi\left(x_{n}\right) q\left(x_{n+1}\right) \ldots q\left(x_{n+m}\right)+u^{i+1} \ell(A)
$$

where $n, m \geq 0$ and $x_{j} \in A$ for all $j$. (If $n$ or $m$ equals zero we have an empty product which equals 1 by definition.) We can describe the relations among these generators. Firstly they are additive in each variable $x_{j}$. Secondly there is a relation corresponding to each of the relations (24)-(27) for example

$$
\begin{aligned}
& u^{i} \phi\left(x_{1}\right) \ldots \phi\left(x_{t}^{\prime} x_{t}^{\prime \prime}\right) \ldots \phi\left(x_{n}\right) q\left(x_{n+1}\right) \ldots q\left(x_{n+m}\right) \\
& \quad=\left(1-\sigma\left(x_{t}^{\prime}\right) \sigma\left(x_{t}^{\prime \prime}\right)\right) u^{i} \phi\left(x_{1}\right) \ldots \phi\left(x_{t}^{\prime}\right) \phi\left(x_{t}^{\prime \prime}\right) \ldots \phi\left(x_{n}\right) q\left(x_{n+1}\right) \ldots q\left(x_{n+m}\right)
\end{aligned}
$$

modulo $u^{i+1} \ell(A)$. If the map $\Phi_{i}$ exists such that the diagram commutes it must send (28) to

$$
u^{i} \otimes \Phi\left(\phi\left(x_{1}\right) \ldots \phi\left(x_{n}\right) q\left(x_{n+1}\right) \ldots q\left(x_{n+m}\right)\right) .
$$

But this formula gives a well defined map by the above identification of the relations among the generators. 
The map $u^{i} \otimes-$ is an isomorphism so if $\Phi$ is also an isomorphism we see that $u^{i}$. is injective. By definition $u^{i}$. is always surjective so the result follows.

Definition 4.7. Let $n \mathrm{~F}_{p}$ denote the category of non-negatively graded $\mathbf{F}_{p^{-}}$ vector spaces. Define the free functor $S_{\mathscr{F}}: n \mathrm{~F}_{p} \rightarrow \mathscr{F}$ to be the left adjoint of the forgetful functor $\mathscr{F} \rightarrow n \mathrm{~F}_{p}$.

REMARK 4.8. We have $S_{\mathscr{F}}(V \oplus W)=S_{\mathscr{F}}(V) \otimes S_{\mathscr{F}}(W)$. Furthermore there is an explicit description as follows

$$
S_{\mathscr{F}}(V)=S_{\mathscr{A} l g}\left(V \oplus \beta V^{* \geq 1} \oplus \bigoplus_{i \geq 1, v \in\{0,1\}} \beta^{v} \lambda^{i}\left(\beta V^{\text {even, }, \geq 2} \oplus V^{\text {odd }, * \geq 2}\right)\right)
$$

where $S_{\mathscr{A l} g}$ denotes the left adjoint of the forgetful functor $\mathscr{A} l g \rightarrow n \mathrm{~F}_{p}$.

TheOREM 4.9. The map $\Phi: \widetilde{\Omega}(A) \rightarrow H^{*}(\bar{\Omega}(A))$ is an isomorphism when A is a free object in $\mathscr{F}$.

Proof. By the results in the appendix section 10 it suffices to show that $\Phi$ is an isomorphism when $A=F_{n}=S_{\mathscr{F}}\left(V_{n}\right), n \geq 0$ where $V_{n}$ is the free $\mathrm{F}_{p}$-vector space on one single generator $x_{n}$ of degree $n$.

We have $F_{0}=\mathrm{F}_{p}\left[x_{0}\right] /\left(x_{0}^{p}-x_{0}\right)$ and $\bar{\Omega}\left(F_{0}\right)=F_{0}$ with zero differential such that $H^{*}\left(\bar{\Omega}\left(F_{0}\right)\right)=F_{0}$. On the other hand $\widetilde{\Omega}\left(F_{0}\right) \cong F_{0}$ with generator $\phi\left(x_{0}\right)$. So $\Phi$ is an isomorphism since $\Phi\left(\phi\left(x_{0}\right)\right)=x_{0}^{p}=x_{0}$.

Further, $F_{1}=\Lambda\left(x_{1}\right) \otimes \mathrm{F}_{p}\left[\beta x_{1}\right]$ with $\lambda x_{1}=x_{1}$. Since $\left(d x_{1}\right)^{p}=d x_{1}$ we can use the idempotents from Remark 4.11 below to get a splitting

$$
\bar{\Omega}\left(F_{1}\right)=\bigoplus_{i \in \mathrm{F}_{p}} e_{i} \bar{\Omega}\left(F_{1}\right)
$$

For each $i$ we have $d e_{i}=0$ and $\left(d x_{1}\right) e_{i}=i e_{i}$. Also $d \beta x_{1}=d \beta \lambda x_{1}=0$. Thus $d\left(x_{1}^{\epsilon}\left(\beta x_{1}\right)^{r} e_{i}\right)=\epsilon i\left(\beta x_{1}\right)^{r} e_{i}$. It follows that $H^{*}\left(e_{i} \bar{\Omega}\left(F_{1}\right)\right)=0$ for $i \neq 0$ and $H^{*}\left(e_{0} \bar{\Omega}\left(F_{1}\right)\right)=F_{1}$ such that $H^{*}\left(\bar{\Omega}\left(F_{1}\right)\right)=F_{1}$. Since $\Phi\left(\phi\left(x_{1}\right)\right)=x_{1} e_{0}$ and $\Phi\left(q\left(x_{1}\right)\right)=\beta x_{1}$ we see that $\Phi$ is surjective. The relations $\phi\left(\beta x_{1}\right)=q\left(x_{1}\right)^{p}$ and $q\left(\beta x_{1}\right)=0$ shows that $\phi\left(x_{1}\right)$ and $q\left(x_{1}\right)$ generate $\widetilde{\Omega}(K)$ so $\Phi$ is also injective.

Assume that $n$ is even and $n \geq 2$. In the following we write [-] for the functor which takes a set to the vector space it generates. We have

$$
F_{n}=S_{\mathscr{A l g}}\left[x_{n}, \beta x_{n}, \lambda^{i} \beta x_{n}, \beta \lambda^{i} \beta x_{n} \mid i \geq 1\right]
$$

and we find that $\bar{\Omega}\left(F_{n}\right)=F_{n} \otimes S_{\mathscr{A l g}}\left[d x_{n}, d \beta x_{n}\right]$. We change basis such that 
the differential becomes easier to describe:

$$
\begin{aligned}
\bar{\Omega}\left(F_{n}\right)=S_{\mathscr{A l g}}\left[x_{n}, d x_{n}\right] \otimes S_{\mathscr{A l g}}\left[\beta x_{n}, d \beta x_{n}\right] \\
\\
\otimes S_{\mathscr{A l g}}\left[\lambda^{i} \beta x_{n}-\left(d \lambda^{i-1} \beta x_{n}\right)^{p-1} \lambda^{i-1} \beta x_{n}, \beta \lambda^{i} \beta x_{n} \mid i \geq 1\right] .
\end{aligned}
$$

By the Künneth formula we find that $H^{*}\left(\bar{\Omega}\left(F_{n}\right)\right)$ equals

$$
S_{\mathscr{A l g}}\left[x_{n}^{p}, x_{n}^{p-1} d x_{n}\right] \otimes S_{\mathscr{A l g}}\left[\lambda^{i} \beta x_{n}-\left(d \lambda^{i-1} \beta x_{n}\right)^{p-1} \lambda^{i-1} \beta x_{n}, \beta \lambda^{i} \beta x_{n} \mid i \geq 1\right] .
$$

The algebra $\widetilde{\Omega}\left(F_{n}\right)$ is generated by the classes $\phi\left(x_{n}\right), \phi\left(\lambda^{i} \beta x_{n}\right), q\left(x_{n}\right)$ and $q\left(\lambda^{i} \beta x_{n}\right)$ where $i \geq 0$. We see that $\Phi$ maps these generators to the free generators for the cohomology of $\bar{\Omega}\left(F_{n}\right)$. Hence $\Phi$ is an isomorphism. The case where $n$ is odd and $n \geq 3$ is similar.

LEMMA 4.10. There is an isomorphism of rings as follows

$$
\alpha: \mathrm{F}_{p}[x] /\left(x^{p}-x\right) \rightarrow\left(\mathrm{F}_{p}\right)^{p} ; \quad x \mapsto(0,1,2, \ldots, p-1)
$$

where $\mathrm{F}_{p}[x]$ is the polynomial ring in one variable $x$ of degree zero and $\left(\mathrm{F}_{p}\right)^{p}$ is the p-fold Cartesian product of $\mathrm{F}_{p}$ by itself.

Proof. Use the factorization $x^{p}-x=\prod_{n \in \mathrm{F}_{p}}(x-n)$ and the Chinese remainder theorem.

Remark 4.11. Let $e_{n}=\alpha^{-1}(0, \ldots, 0,1,0, \ldots, 0)$ with the 1 on the $n$th place for $n \in \mathrm{F}_{p}$. Clearly $e_{n} e_{m}=0$ for $n \neq m, e_{n}^{2}=e_{n}$ and $\sum e_{n}=1$. Also $x e_{n}=n e_{n}$. Finding eigenvectors for $x f(x)=n f(x)$ and normalizing one gets the following:

$$
e_{0}=1-x^{p-1}, \quad e_{m}=-\sum_{i=1}^{p-1}\left(\frac{x}{m}\right)^{i}, \quad m \neq 0 .
$$

\section{Steenrod diagonal elements}

In this section we use the functor $R_{+}$of [6] to define a functor $R: \mathscr{K} \rightarrow \mathscr{K}$. We need $R$ for a description of $\ell$ given in the next section. Let $K$ denote an unstable $\mathscr{A}$-algebra and consider $\mathrm{F}_{p}[u]$ with $|u|=2$ an object in $\mathscr{K}$ by the isomorphism $\mathrm{F}_{p}[u] \cong H^{*}(B \mathrm{~T})$. by

Definition 5.1. For $x \in K$ and $\epsilon=0,1$ we define $S t_{\epsilon}(x) \in \mathrm{F}_{p}[u] \otimes K$

$$
S t_{\epsilon}(x)=u^{-\epsilon \hat{\sigma}(x)} \sum_{i \geq 0}\left(-u^{p-1}\right)^{[|x| / 2]-i} \otimes \beta^{\epsilon} P^{i} x .
$$


Note that the terms where the total exponent of $u$ is negative has $\beta^{\epsilon} P^{i} x=0$. Let $R(K) \subseteq \mathrm{F}_{p}[u] \otimes K$ be the sub- $\mathrm{F}_{p}$-algebra generated by $u \otimes 1$ and $S t_{\epsilon}(x)$ for all $x \in K$ and $\epsilon=0,1$.

TheORem 5.2. For each $\theta \in \mathscr{A}$ one has $\theta R(K) \subseteq R(K)$. Thus $R$ is a functor $R: \mathscr{K} \rightarrow \mathscr{K}$. The explicit formulas are as follows where $n=[|x| / 2]$ and $\epsilon=0,1$ :

$$
\begin{gathered}
P^{i} S t_{\epsilon}(x)=\sum_{t}\left(\begin{array}{c}
(p-1)(n-t)+\epsilon \sigma(x) \\
i-p t
\end{array}\right) u^{(p-1)(i-p t)} S t_{\epsilon}\left(P^{t} x\right) \\
-\epsilon(-1)^{\sigma(x)} \sum_{t}\left(\begin{array}{c}
(p-1)(n-t)-1+\sigma(x) \\
i-p t-1
\end{array}\right) \\
\cdot u^{(p-1)(i-p t)-1+(2-p) \sigma(x)} S t_{0}\left(\beta P^{t} x\right), \\
\beta S t_{\epsilon}(x)=(1-\epsilon) u^{\hat{\sigma}(x)} \operatorname{St}_{1}(x) .
\end{gathered}
$$

Proof. The formula for the Bockstein operation follows directly by the definition of $S t_{\epsilon}(x)$. We use results from [6] to prove the other formula. By [13] we have that $\mathrm{F}_{p}\left[u, u^{-1}\right]$ is an $\mathscr{A}$-algebra with $\beta=0$ and

$$
P^{i} u^{j}=\left(\begin{array}{l}
j \\
i
\end{array}\right) u^{j+i(p-1)} ; \quad i, j \in \mathrm{Z} ; \quad i \geq 0 .
$$

Here the following extended definition of binomial coefficients is used where $r \in \mathrm{R}$ and $k \in \mathrm{Z}$.

$$
\left(\begin{array}{l}
r \\
k
\end{array}\right)= \begin{cases}\frac{r(r-1) \ldots(r-k+1)}{k !}, & k>0 \\
1, & k=0 \\
0, & k<0\end{cases}
$$

Let $\Delta=\Lambda(a) \otimes \mathrm{F}_{p}\left[b, b^{-1}\right]$ with $|a|=2 p-3,|b|=2 p-2$ be the $\mathscr{A}$-algebra introduced in [6] (2.6). That is $\beta a=b$ and

$$
\begin{gathered}
P^{i}\left(b^{j}\right)=(-1)^{i}\left(\begin{array}{c}
(p-1) j \\
i
\end{array}\right) b^{i+j} \\
P^{i}\left(a b^{j-1}\right)=(-1)^{i}\left(\begin{array}{c}
(p-1) j-1 \\
i
\end{array}\right) a b^{i+j-1} .
\end{gathered}
$$


Note that we have changed the names of the generators. In [6] they were named $u$ and $v$ instead of $a$ and $b$. We define an additive transfer map as follows:

$$
\tau: \Delta \rightarrow \mathrm{F}_{p}\left[u, u^{-1}\right] ; \quad b^{j} \mapsto 0 ; \quad a b^{j-1} \mapsto\left(-u^{p-1}\right)^{j} u^{-1} .
$$

Note that $|\tau|=-1$. A direct verification shows that $\tau$ is $\mathscr{A}$-linear.

A functor $R_{+}$from the category of graded $\mathscr{A}$-modules to itself is constructed in [6]. In the case of an unstable $\mathscr{A}$-algebra $K$ it comes with an $\mathscr{A}$-linear map $f: R_{+} K \rightarrow \Sigma \Delta \otimes K$ defined by [6] (3.1), (3.2). The composite

$$
R_{+} K \stackrel{f}{\longrightarrow} \sigma \Delta \otimes K \stackrel{\Sigma \tau \otimes 1}{\longrightarrow} \Sigma \mathrm{F}_{p}\left[u, u^{-1}\right] \otimes K
$$

is given by

$$
\begin{aligned}
s b^{k} \otimes x & \mapsto-s \sum_{j}\left(-u^{p-1}\right)^{k-j} u^{-1} \otimes \beta P^{j} x, \\
s a b^{k-1} \otimes x & \mapsto s \sum_{j}\left(-u^{p-1}\right)^{k-j} u^{-1} \otimes P^{j} x .
\end{aligned}
$$

Especially $s b^{n} \otimes x \mapsto-s u^{\sigma(x)} S t_{1}(x)$ and $s a b^{n-1} \otimes x \mapsto s u^{-1} S t_{0}(x)$ where $n=[|x| / 2]$. The formulas [6] (3.4), (3.5) for the $\mathscr{A}$-action on $R_{+} M$ gives the following formulas for the $\mathscr{A}$-action on $u^{\sigma(x)} S t_{1}(x)$ and $u^{-1} S t_{0}(x)$ :

$$
\begin{gathered}
P^{i}\left(u^{\sigma(x)} S t_{1}(x)\right)=\sum_{t}\left(\begin{array}{c}
(p-1)(n-t) \\
i-p t
\end{array}\right) u^{(p-1)(i-p t)-\sigma(x)} S t_{1}\left(P^{t} x\right) \\
-\sum_{t}(-1)^{\sigma(x)}\left(\begin{array}{c}
(p-1)(n-t)-1 \\
i-p t-1
\end{array}\right) \\
\cdot u^{(p-1)(i-p t-\sigma(x))-1} S t_{0}\left(\beta P^{t} x\right), \\
P^{i}\left(u^{-1} S t_{0}(x)\right)=\sum_{t}\left(\begin{array}{c}
(p-1)(n-t)-1 \\
i-p t
\end{array}\right) u^{(p-1)(i-p t)-1} S t_{0}\left(P^{t} x\right) .
\end{gathered}
$$

This proves the result directly for $\sigma(x)=0$ and $\epsilon=1$. By the Cartan formula applied to $u u^{-1} S t_{\epsilon}(x)$ we have that $P^{i} S t_{\epsilon}(x)=u P^{i}\left(u^{-1} S t_{\epsilon}(x)\right)+$ $u^{p} P^{i-1}\left(u^{-1} S t_{\epsilon}(x)\right)$. By combining this with the formulas above we get the result in the other cases.

\section{A pullback description of the functor $\ell$}

In this section we describe $\ell(K)$ as a pullback in the case where $K$ is a free object in $\mathscr{K}$. We start by a result on cohomology of Eilenberg-MacLane spaces. 
Recall that a sequence of integers $I=\left(\epsilon_{1}, s_{1}, \epsilon_{2}, s_{2}, \ldots, \epsilon_{k}, s_{k}, \epsilon_{k+1}\right)$ with $s_{i} \geq 0$ and $\epsilon_{i} \in\{0,1\}$ is called admissible if $s_{i} \geq p s_{i+1}+\epsilon_{i+1}$ and $s_{k} \geq 1$ or if $k=0$ when $I=(\epsilon)$. The degree of $I$ is defined as $|I|=\sum \epsilon_{j}+\sum 2 s_{j}(p-1)$ and the excess is defined recursively by $e((\epsilon, s), J)=2 s+\epsilon-|J|$. We use the following notation $P^{I}=\beta^{\epsilon_{1}} P^{s_{1}} \beta^{\epsilon_{2}} P^{s_{2}} \ldots \beta^{\epsilon_{k}} P^{s_{k}} \beta^{\epsilon_{k+1}}$.

LemMa 6.1. The cohomology ring of the Eilenberg-MacLane space $K\left(\mathrm{~F}_{p}, n\right)$ can be written in the following form when $n \geq 2$ :

$$
H^{*}\left(K\left(\mathrm{~F}_{p}, n\right)\right)=S_{\mathscr{F}}\left[P^{I} \iota_{n} \mid I \text { is admissible, } e(I) \leq n-2, \epsilon_{1}=0\right] .
$$

Furthermore, $H^{*}\left(K\left(\mathrm{~F}_{p}, 1\right)\right)=S_{\mathscr{F}}\left[\iota_{1}\right]$ and $H^{*}\left(K\left(\mathrm{~F}_{p}, 0\right)\right)=S_{\mathscr{F}}\left[\iota_{0}\right]$.

Proof. The cases $n=0,1$ are trivial. Assume that $n \geq 2$ and define the set

$$
A(n)=\{I \mid I \text { is admisseble, } e(I) \leq n-1,|I|+n \text { is odd }\} .
$$

Remark that if $I \in A(n)$ then $(0,(|I|+n-1) / 2, I) \in A(n)$. To see this write $I \in A(n)$ as $I=\left(\epsilon, s, I^{\prime}\right)$. Then $e(I)=2 s+\epsilon-\left|I^{\prime}\right| \leq n-1$ or equivalently $2 s p+2 \epsilon-|I| \leq n-1$ such that the sequence $(0,(|I|+n-1) / 2, I)$ is admissible. Its excess is $n-1$ and its degree plus $n$ is odd since $p-1$ is even.

By Cartan's computation (a special case of [9], Theorem 10.3) we have that $H^{*} B^{n} \mathrm{~F}_{p}$ is the free graded commutative algebra on the set

$$
B=\left\{P^{J} \iota_{n} \mid J \text { is admissible, } e(J)<n \text { or }\left(e(J)=n \text { and } \epsilon_{1}=1\right)\right\} .
$$

Assume that $P^{I} \iota_{n}$ belongs to the set in the statement of the lemma. Then $P^{I} \iota_{n}$ and $\beta P^{I} \iota_{n}$ belongs to $B$. By the remark we see that if $|I|+n$ is even then $\beta^{\epsilon} \lambda^{i} \beta P^{I} \iota_{n} \in B$ and if $|I|+n$ is odd then $\beta^{\epsilon} \lambda^{i} P^{I} \iota_{n} \in B$ for $\epsilon=0,1$ and $i \geq 1$.

Conversely, assume that $P^{J} \iota_{n} \in B$. If $e(J) \leq n-2$ or $e(J)=n-1$ and $\epsilon_{1}=1$ it is clearly one of the generators described in the lemma. It suffices to handle the case $e(J)=n-1, \epsilon_{1}=0$ since the case $e(J)=n, \epsilon_{1}=1$ then follows. Write $J$ as $J=\left(0, s, J^{\prime}\right)$ where $e(J)=2 s-|J|^{\prime}=n-1$. Then $2 s=n+\left|J^{\prime}\right|-1$ such that $P^{J} \iota_{n}=\lambda P^{J^{\prime}} \iota_{n}$ and $e(J) \leq e\left(J^{\prime}\right)$. We can continue this process until the next $\epsilon$ equals one or the excess drops below $n-1$.

Proposition 6.2. For any object $K$ in $\mathscr{K}$ there is natural morphism of $\mathrm{F}_{p}$-algebras $\Delta: \ell(K) \rightarrow \mathrm{F}_{p}[u] \otimes K$ defined by

$$
\phi(x) \mapsto S t_{0}(x), \quad q(x) \mapsto S t_{1}(x), \quad \delta(x) \mapsto 0, \quad u \mapsto u \otimes 1 .
$$

The image of this morphism is $\operatorname{Im}(\Delta)=R(K)$. 
Proof. We check that (10)-(21) are mapped to zero by the formulas defining $\Delta$. Since $\delta(x)$ is mapped to zero this is trivial for all elements except (14), (15), (16) and (20). that

By the Cartan formula and $\left[\frac{|a b|}{2}\right]=\left[\frac{|a|}{2}\right]+\left[\frac{|b|}{2}\right]+\sigma(a) \sigma(b)$ one verifies

$$
\begin{aligned}
& S t_{0}(a b)=\left(-u^{p-1}\right)^{\sigma(a) \sigma(b)} S t_{0}(a) S t_{0}(b) \\
& S t_{1}(a b)=\left(-u^{p-1}\right)^{\sigma(a) \sigma(b)}\left(u^{\sigma(b)} S t_{1}(a) S t_{0}(b)+(-u)^{\sigma(a)} S t_{0}(a) S t_{1}(b)\right)
\end{aligned}
$$

such that (14) and (15) are mapped to zero. Lemma 2.7 implies that (16) and (20) are mapped to zero.

Proposition 6.3. If $K$ is a free object in $\mathscr{K}$ then $\operatorname{ker}(\Delta)=I_{\delta}(K)$.

Proof. Assume that $K=S_{\mathscr{K}}(V)$ for a non negatively graded vector space $V$. We must show that $\bar{\Delta}: \ell(K) / I_{\delta}(K) \rightarrow \mathrm{F}_{p}[u] \otimes K$ is injective.

The algebra $\ell(K) / I_{\delta}(K)$ has generators $\phi(x), q(x)$ for $x \in K$ and $u$. The relations are that $\phi$ and $q$ are additive and that (14), (15), (16) and (20) equals zero. Let $\left\{v_{s} \mid s \in S\right\}$ denote a basis for $V$. By Lemma 6.1 we find that $K=S_{\mathscr{F}}(W)$ where $W$ is the graded vector space with basis

$$
B=\left\{P^{I} v_{s} \mid I \text { admissible, } e(I) \leq\left|v_{s}\right|-2, \epsilon_{1}=0, s \in S\right\} .
$$

We see that the following elements are algebra generators for $\ell(K) / I_{\delta}(K)$ where $a \in B^{0}, b \in B^{1}, v \in B^{\text {odd, } * \geq 3}, w \in B^{\text {even, } * \geq 2}$ and $i \geq 0$ :

$$
\begin{gathered}
u, \phi(a), \phi(b), q(\beta b), \\
\phi(\beta v), \phi\left(\lambda^{i} v\right), q(\beta v), q\left(\lambda^{i} v\right), \\
\phi(w), \phi\left(\lambda^{i} \beta w\right), q(w), q\left(\lambda^{i} \beta w\right) .
\end{gathered}
$$

We claim that these generators are mapped to algebraically independent elements in $\mathrm{F}_{p}[u] \otimes K$. By the formulas defining $\Delta$ we see that it suffices to check this claim in the case where $V$ is one dimensional. So assume that $K=S_{\mathscr{K}}\left[\iota_{n}\right]$ where $\left|\iota_{n}\right|=n$.

For any $n$ we have $u \mapsto u \otimes 1$. For $n=0$ we have $\phi\left(\iota_{0}\right) \mapsto 1 \otimes \iota_{0}$ and for $n=1$ we have $\phi\left(\iota_{1}\right) \mapsto 1 \otimes \iota_{1}, q\left(\iota_{1}\right) \mapsto 1 \otimes \beta \iota_{1}$ so in these two cases the claim holds.

Assume that $n \geq 2$. The algebra generators are mapped as follows modulo 
elements in the ideal $\left(u^{p-1} \otimes 1\right)$ :

$$
\begin{aligned}
\phi(\beta v) & \mapsto 1 \otimes(\beta v)^{p}, & q(\beta v) & \mapsto-u^{p-2} \otimes \beta P^{(|v|-1) / 2} \beta v, \\
\phi\left(\lambda^{i} v\right) & \mapsto 1 \otimes \lambda^{i+1} v, & q\left(\lambda^{i} v\right) & \mapsto 1 \otimes \beta \lambda^{i+1} v, \\
\phi(w) & \mapsto 1 \otimes w^{p}, & q(w) & \mapsto-u^{p-2} \otimes \beta P^{|w| / 2-1} w, \\
\phi\left(\lambda^{i} \beta w\right) & \mapsto 1 \otimes \lambda^{i+1} \beta w, & q\left(\lambda^{i} \beta w\right) & \mapsto 1 \otimes \beta \lambda^{i+1} \beta w .
\end{aligned}
$$

If $|I|+n$ is odd we must look closer at $\beta P^{(|I|+n-1) / 2} \beta P^{I} \iota_{n}$. Write $I$ as $I=\left(0, s, I^{\prime}\right)$. We have $e(I)=2 s-\left|I^{\prime}\right| \leq n-2$ which implies that $\left(0,(|I|+n-1) / 2,1, s, I^{\prime}\right)$ is admissible. Its excess equals $n-2$ and we see that $P^{(|I|+n-1) / 2} \beta P^{I} \iota_{n} \in B^{\text {even }}$.

If $|I|+n$ is even we must look at $\beta P^{(|I|+n-2) / 2} P^{I} \iota_{n}$. As in the odd case we see that $P^{(|I|+n-2) / 2} P^{I} \iota_{n} \in B^{\text {even }}$. However there is no $\beta$ between the first two $P$-operations from the left.

We conclude that the claim holds for $n \geq 2$ which completes the proof.

In the following $K$ denotes an object in $\mathscr{K}$. Before stating the main theorem we need some definitions and lemmas.

Lemma 6.4. Let $a_{1}, \ldots, a_{p} \in K$ be elements of odd degree and define the following element in $I_{\delta}(K)$ :

$$
D\left(a_{1}, \ldots, a_{p}\right)=\sum_{i=2}^{p} \delta\left(a_{1} a_{i}\right) \delta\left(a_{2}\right) \ldots \widehat{\delta\left(a_{i}\right)} \ldots \delta\left(a_{p}\right) .
$$

where the hat means that the factor is left out. Then for any permutation $\tau \in \Sigma_{p}$ one has $D\left(a_{1}, \ldots, a_{p}\right)=D\left(a_{\tau(1)}, \ldots, a_{\tau(p)}\right)$. The element is mapped as follows under the map $Q: \ell(K) \rightarrow \bar{\Omega}(K)$ :

$$
D\left(a_{1}, \ldots, a_{p}\right) \mapsto \sum_{i=1}^{p} a_{i} d a_{1} \ldots \widehat{d a}_{i} \ldots d a_{p} .
$$

Proof. We first show the invariance under permutation. Since the degree of $\delta\left(a_{i}\right)$ is even $D\left(a_{1}, \ldots, a_{p}\right)$ is invariant under permutations fixing $a_{1}$. Thus it suffices to show that $D\left(a_{1}, a_{2}, \ldots, a_{p}\right)=D\left(a_{2}, \ldots, a_{p}, a_{1}\right)$. We prove the following more general formula for $n \geq 3$ :

$$
\begin{aligned}
& \sum_{i=2}^{n} \delta\left(a_{1} a_{i}\right) \delta\left(a_{2}\right) \ldots \widehat{\delta\left(a_{i}\right)} \ldots \delta\left(a_{n}\right) \\
& =\sum_{j=3}^{n} \delta\left(a_{2} a_{j}\right) \delta\left(a_{1}\right) \delta\left(a_{3}\right) \ldots \widehat{\delta\left(a_{j}\right)} \ldots \delta\left(a_{n}\right)-(n-1) \delta\left(a_{2} a_{1}\right) \delta\left(a_{3}\right) \ldots \delta\left(a_{n}\right) .
\end{aligned}
$$


The proof is by induction on $n$. For $n=3$ we have

$$
\begin{aligned}
\delta\left(a_{1} a_{2}\right) \delta\left(a_{3}\right)+\delta\left(a_{1} a_{3}\right) \delta\left(a_{2}\right) & =\delta\left(a_{1} a_{2}\right) \delta\left(a_{3}\right)-\delta\left(a_{3} a_{1}\right) \delta\left(a_{2}\right) \\
& =2 \delta\left(a_{1} a_{2}\right) \delta\left(a_{3}\right)+\delta\left(a_{2} a_{3}\right) \delta\left(a_{1}\right) \\
& =-2 \delta\left(a_{2} a_{1}\right) \delta\left(a_{3}\right)+\delta\left(a_{2} a_{3}\right) \delta\left(a_{1}\right)
\end{aligned}
$$

where we used (13) at the second equality sign. Assume that the formula holds for $n-1$. Then we have

$$
\begin{aligned}
& \sum_{i=2}^{n} \delta\left(a_{1} a_{i}\right) \delta\left(a_{2}\right) \ldots \widehat{\delta\left(a_{i}\right)} \ldots \delta\left(a_{n}\right) \\
& =\left(\sum_{i=2}^{n-1} \delta\left(a_{1} a_{i}\right) \delta\left(a_{2}\right) \ldots \widehat{\delta\left(a_{i}\right)} \ldots \delta\left(a_{n-1}\right)\right) \delta\left(a_{n}\right)+\delta\left(a_{1} a_{n}\right) \delta\left(a_{2}\right) \ldots \delta\left(a_{n-1}\right) \\
& =\left(\sum_{j=3}^{n-1} \delta\left(a_{2} a_{j}\right) \delta\left(a_{1}\right) \delta\left(a_{3}\right) \ldots \widehat{\delta\left(a_{j}\right)} \ldots \delta\left(a_{n-1}\right)\right) \delta\left(a_{n}\right) \\
& \quad-(n-2) \delta\left(a_{2} a_{1}\right) \delta\left(a_{3}\right) \ldots \delta\left(a_{n-1}\right) \delta\left(a_{n}\right)+\delta\left(a_{1} a_{n}\right) \delta\left(a_{2}\right) \ldots \delta\left(a_{n-1}\right) .
\end{aligned}
$$

Since $\delta\left(a_{1} a_{n}\right) \delta\left(a_{2}\right)+\delta\left(a_{2} a_{1}\right) \delta\left(a_{n}\right)=\delta\left(a_{2} a_{n}\right) \delta\left(a_{1}\right)$ by relation (13) the sum of the last two terms above equals

$$
-(n-1) \delta\left(a_{2} a_{1}\right) \delta\left(a_{3}\right) \ldots \delta\left(a_{n}\right)+\delta\left(a_{2} a_{n}\right) \delta\left(a_{1}\right) \ldots \delta\left(a_{n-1}\right)
$$

and we recover the formula for $n$.

We use that $d\left(a_{1} a_{i}\right)=a_{i} d a_{1}-a_{1} d a_{i}$ to compute the image under $Q$ :

$$
\begin{aligned}
D\left(a_{1}, \ldots, a_{p}\right) & \mapsto \sum_{i=2}^{p} d\left(a_{1} a_{i}\right) d a_{2} \ldots \widehat{d a}_{i} \ldots d a_{p} \\
& =\sum_{i=2}^{p} a_{i} d a_{1} \ldots \widehat{d a}_{i} \ldots d a_{p}-(p-1) a_{1} d a_{2} \ldots d a_{p} .
\end{aligned}
$$

Definition 6.5. For any non negative integer $n$ we let $B(n)$ denote the following set:

$$
B(n)=\left\{\left(\beta_{1}, \ldots, \beta_{p}\right) \in \mathrm{Z}^{p} \mid \forall i: \beta_{i} \geq 0, \beta_{1}+\cdots+\beta_{p}=n, \exists i, j: \beta_{i} \neq \beta_{j}\right\} .
$$

The cyclic group on $p$ elements $C_{p}$ act on $B(n)$ by cyclic permutation of 
coordinates. For $x \in K$ we define the following elements in $I_{\delta}(K)$ :

$$
\begin{aligned}
& D_{0}^{n}(x)=-\sigma(x) \sum D\left(P^{\beta_{1}}(x), P^{\beta_{2}}(x), \ldots, P^{\beta_{p}}(x)\right), \\
& D_{1}^{n}(x)=\hat{\sigma}(x) \sum \delta\left(P^{\beta_{1}}(x) P^{\beta_{2}}(x) \ldots P^{\beta_{p}}(x)\right)
\end{aligned}
$$

where both sums are taken over $\beta \in B(n) / C_{p}$. Note that $D_{0}^{n}(x)$ is well defined by Lemma 6.4

Lemma 6.6. For any $x \in K$ the following formulas hold in $\bar{\Omega}(K)$ :

$$
\begin{aligned}
& P^{i} \circ Q(\phi(x))=Q\left(\phi\left(P^{i / p} x\right)+D_{0}^{i}(x)\right), \\
& P^{i} \circ Q(q(x))=Q\left(q\left(P^{i / p} x\right)+D_{1}^{i}(x)\right)
\end{aligned}
$$

where by convention $P^{t}=0$ when $t$ is a rational number which is not a non negative integer.

Proof. We first prove (29). Recall that $Q(\phi(x))=x^{p}+\lambda x-x(d x)^{p-1}$. We have $P^{i} \lambda x=\lambda P^{i / p} x$ by Lemma 2.7 and also $P^{i}\left(x^{p}\right)=\left(P^{i / p} x\right)^{p}$ so it suffices to prove the following for $|x|$ odd:

$$
P^{i}\left(x(d x)^{p-1}\right)=\left(P^{i / p} x\right)\left(d P^{i / p} x\right)^{p-1}-Q\left(D_{0}^{i}(x)\right) .
$$

By the Cartan formula we have

$$
P^{i}\left(x(d x)^{p-1}\right)=\sum P^{\beta_{1}}(x) d P^{\beta_{2}}(x) \ldots d P^{\beta_{p}}(x)
$$

where we sum over the tuples $\left(\beta_{1}, \ldots, \beta_{p}\right)$ with $\sum \beta_{j}=i$. The cyclic group $C_{p}$ acts on the set of such tuples and an orbit has length 1 or $p$. Arranging the terms according to this the result follows by the definition of $D_{0}^{i}(x)$ and Lemma 6.4.

For the proof of (30) recall that $Q(q(x))=x^{p-1} d x+\beta \lambda x$. We have $P^{i}(\beta \lambda x)=\beta \lambda\left(P^{i / p} x\right)+\left(\beta P^{(i-1) / p} x\right)^{p}$ by Lemma 2.7 so when $|x|$ is odd we are done. For $|x|$ even we must show that

$$
P^{i}\left(x^{p-1} d x\right)=\left(P^{i / p} x\right)^{p-1} d P^{i / p} x+Q\left(D_{1}^{i}(x)\right) .
$$

This follows by the Cartan formula and a similar argument on orbits as the above.

THEOREM 6.7. For any object $K$ in $\mathscr{K}$ there is an $\mathscr{A}$-module structure on $\ell(K)$ such that $\ell$ becomes a functor $\ell: \mathscr{K} \rightarrow \mathscr{K}$. The explicit formulas for the 
action are as follows where $x \in K, n=[|x| / 2]$ and $i \geq 0$. Firstly, the action on $\phi(x)$ is given by:

$$
\begin{aligned}
P^{i} \phi(x) & =D_{0}^{i}(x)+\sum_{t}\left(\begin{array}{c}
(p-1)(n-t) \\
i-p t
\end{array}\right) u^{(p-1)(i-p t)} \phi\left(P^{t} x\right), \\
\beta \phi(x) & =u^{\hat{\sigma}(x)}\left(q(x)-\delta(x)^{p-2} \delta(x \beta x)\right) .
\end{aligned}
$$

Secondly, the action on $q(x)$ is given by:

$$
\begin{gathered}
P^{i} q(x)=D_{1}^{i}(x)+\sum_{t}\left(\begin{array}{c}
(p-1)(n-t)+\sigma(x) \\
i-p t
\end{array}\right) u^{(p-1)(i-p t)} q\left(P^{t} x\right) \\
-(-1)^{\sigma(x)} \sum_{t}\left(\begin{array}{c}
(p-1)(n-t)-1+\sigma(x) \\
i-p t-1
\end{array}\right) \\
\cdot u^{(p-1)(i-p t)-1+(2-p) \sigma(x)} \phi\left(\beta P^{t} x\right), \\
\beta q(x)=-\delta\left(x^{p-1} \beta x\right) .
\end{gathered}
$$

Thirdly, the actions on $\delta(x)$ and $u$ are as follows:

$$
P^{i} \delta(x)=\delta\left(P^{i} x\right), \quad \beta \delta(x)=-\delta(\beta x), \quad P^{1} u=u^{p}, \quad \beta u=0 .
$$

Furthermore the maps $Q$ and $\Delta$ becomes $\mathscr{A}$-linear and there is a commutative diagram in $\mathscr{K}$ as follows:

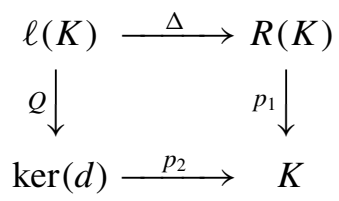

where the morphisms $p_{1}$ and $p_{2}$ are given by $p_{1}(u)=0, p_{1}(x)=x, p_{2}(d x)=$ $0, p_{2}(x)=x$ for $x \in K$. Finally, if $K$ is a free object in $\mathscr{K}$ then the diagram is a pullback square.

Proof. By the definition of $\Delta$ and $Q$ there is a commutative diagram as stated in the category of $\mathrm{F}_{p}$-algebras. We first prove that this diagram is a pullback when $K$ is a free object in $\mathscr{K}$.

By Lemma 6.1 and Theorem 4.9 the map $\Phi$ is an isomorphism. So by Theorem 4.5 the kernel of $Q$ is the ideal $(u) \subseteq \ell(K)$. The kernel of $p_{1}$ is the ideal $(u \otimes 1) \subseteq R(K)$ so it suffices to show that the restriction of the map $\Delta$ to these kernels $\Delta \mid:(u) \rightarrow(u \otimes 1)$ is an isomorphism. It is surjective since $\Delta$ is surjective and $\Delta(u)=u \otimes 1$. By Proposition 6.3 we have $\operatorname{ker}(\Delta)=I_{\delta}(K)$ 
such that $\operatorname{ker}(\Delta \mid)=(u) \cap I_{\delta}(K)$. Let $x \in(u) \cap I_{\delta}(K)$. We can write $x=u z$ for some $z \in \ell(K)$. Since $0=\Delta(u z)=u \Delta(z)$ we have $\Delta(z)=0$ so $z \in I_{\delta}(K)$ and $x=u z=0$. Thus $(u) \cap I_{\delta}(K)=0$ and $\Delta \mid$ is injective.

When $K$ is a free object the pullback defines an $\mathscr{A}$-module structure on $\ell(K)$. By Theorem 5.2 and Lemma 6.6 we see that the stated formulas describe this $\mathscr{A}$-action. A standard naturality argument now proves the statements for general objects $K$ in $\mathscr{K}$.

\section{Homotopy orbits of T-spaces}

In this section we list some results on homotopy orbits of T-spaces. They are all similar to results for $p=2$ considered in [2] and we often refer to the proofs given there. In the entire section $Y$ denotes a T-space. We write $C_{n}$ for the cyclic group of order $n$. We let $u$ of degree $|u|=2$ and $v$ of degree $|v|=1$ denote algebra generators as follows: $H^{*} \mathrm{~T}=\Lambda(v), H^{*} B \mathrm{~T}=\mathrm{F}_{p}[u]$ and $H^{*} B C_{p^{n}}=\Lambda(v) \otimes \mathrm{F}_{p}[u]$.

Proposition 7.1. The fibration $Y \rightarrow E \mathrm{~T} \times_{\mathrm{T}} Y \rightarrow B \mathrm{~T}$ has the following Leray-Serre spectral sequence:

$$
E_{2}^{* *}=H^{*}(B \mathrm{~T}) \otimes H^{*}(Y) \Rightarrow H^{*}\left(E \mathrm{~T} \times_{\mathrm{T}} Y\right) .
$$

The differential in the $E_{2}$-term is given by

$$
d_{2}: H^{*}(Y) \rightarrow u H^{*}(Y) ; \quad d_{2}(y)=u d(y)
$$

where $d$ is the differential associated to the T-action (see Proposition 2.3).

Proof. Similar to the proof of [2] Proposition 3.3.

Definition 7.2. Let $E_{\infty} Y=E \mathrm{~T} \times_{\mathrm{T}} Y$ and define

$$
E_{n} Y=E \mathrm{~T} \times{ }_{C_{p^{n}}} Y \quad \text { for } \quad n=0,1,2, \ldots
$$

For nonnegative integers $n$ and $m$ with $m>n$ define the maps

$$
q_{m}^{n}: H^{*} E_{m} Y \rightarrow H^{*} E_{n} Y, \quad \tau_{n}^{m}: H^{*} E_{n} Y \rightarrow H^{*} E_{m} Y
$$

by letting $q_{m}^{n}$ be the map induced by the quotient map and $\tau_{n}^{m}$ be the transfer map. Also define $q_{\infty}^{n}: H^{*} E_{\infty} Y \rightarrow H^{*} E_{n} Y$ as the map induced by the quotient.

The following theorem is inspired by a result of Tom Goodwillie which can be found in [8] p. 279. 
THEOREM 7.3. There is a commutative diagram as follows for any $m \geq 1$ :

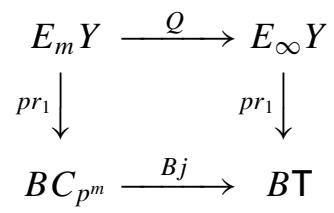

Here $Q$ denotes the quotient map and $j: C_{p^{m}} \hookrightarrow \mathrm{T}$ the inclusion. The diagram gives rise to an isomorphism.

$\Theta: H^{*}\left(B C_{p^{m}}\right) \otimes_{H^{*}(B \mathrm{~T})} H^{*}\left(E_{\infty} Y\right) \cong H^{*}\left(E_{m} Y\right) ; \quad x \otimes y \mapsto p r_{1}^{*}(x) q_{\infty}^{m}(y)$

The transfer map $\tau_{m}^{m+1}: H^{*} E_{m} Y \rightarrow H^{*} E_{m+1} Y$ is zero on elements of the form $\Theta(1 \otimes y)$ and the identity on elements of the form $\Theta(v \otimes y)$. We get an isomorphism

$$
\operatorname{colim} H^{*} E_{m} Y=v H^{*} E_{\infty} Y \cong \widetilde{H}^{*}\left(\Sigma\left(E_{\infty} Y\right)_{+}\right) .
$$

Proof. Similar to the proof of [2] Theorem 4.2.

We use the above theorem to give a convenient definition of the T-transfer:

Definition 7.4. For non negative $n$ the T-transfer $\tau_{n}^{\infty}: H^{*} E_{n} Y \rightarrow H^{*} E_{\infty} Y$ is defined as the following composite:

$$
H^{*} E_{n} Y \longrightarrow \operatorname{colim} H^{*} E_{m} Y \stackrel{v^{-1}}{\longrightarrow} H^{*} E_{\infty} Y .
$$

The colimit is taken over the transfer maps $\tau_{m}^{m+1}$. Note that $\left|\tau_{n}^{\infty}\right|=-1$.

Proposition 7.5. Frobenius reciprocity holds for any $n \geq 0$ :

$$
\tau_{n}^{\infty}\left(q_{\infty}^{n}(x) y\right)=(-1)^{|x|} x \tau_{n}^{\infty}(y) .
$$

Furthermore the following composition formulas hold.

$$
\tau_{0}^{\infty} \circ q_{\infty}^{0}=0, \quad q_{\infty}^{0} \circ \tau_{0}^{\infty}=d
$$

Proof. Similar to the proof of [2] Proposition 4.6, 4.7 and 4.8.

Proposition 7.6. There is always an inclusion $\operatorname{Im}\left(q_{\infty}^{0}\right) \subseteq \operatorname{ker}(d)$. If we have equality $\operatorname{Im}\left(q_{\infty}^{0}\right)=\operatorname{ker}(d)$ then the Leray-Serre spectral sequence of the fibration $Y \rightarrow E \mathrm{~T} \times_{\mathrm{T}} Y \rightarrow B \mathrm{~T}$ collapses at the $E_{3}$-term.

Proof. By Proposition 7.5 we have $d \circ q_{\infty}^{0}=q_{\infty}^{0} \circ \tau_{0}^{\infty} \circ q_{\infty}^{0}=0$. The collapse statement follows by Proposition 7.1. 
Definition 7.7. Put $\zeta_{p}=\exp (2 \pi i / p)$ and define the map

$$
f_{Y}^{\prime}: \mathrm{T} \times Y \rightarrow E \mathrm{~T} \times Y^{p} ; \quad(z, y) \mapsto\left(z e, z y, \zeta_{p} z y, \zeta_{p}^{2} z y, \ldots, \zeta_{p}^{p-1} z y\right) .
$$

We let $C_{p}$ act on the space to the left by $\zeta_{p} \cdot(z, y)=\left(\zeta_{p} z, y\right)$ and on the space to the right by $\zeta_{p} \cdot\left(e, y_{1}, \ldots, y_{p}\right)=\left(\zeta_{p} e, y_{2}, \ldots, y_{p}, y_{1}\right)$. Then the above map is $C_{p}$-equivariant. Passing to the quotients we get a map

$$
f_{Y}: \mathrm{T} / C_{p} \times Y \rightarrow E \mathrm{~T} \times{ }_{C_{p}} Y^{p} .
$$

Note that this map is natural in $Y$ with respect to $C_{p}$-equivariant maps.

Recall the followings facts on the order $p$ cyclic construction [10], [9] and [12]. For any space $X$ with homology of finite type there is a natural isomorphism

$$
H^{*}\left(E \mathrm{~T} \times C_{p} X^{p}\right) \cong H^{*}\left(C_{p} ; H^{*}(X)^{\otimes p}\right)
$$

where $C_{p}$ acts on $H^{*}(X)^{\otimes p}$ by cyclic permutation with the usual sign convention. For a homogeneous element $y \in H^{*} X$ the $C_{p}$ invariant $y^{\otimes p}$ defines an element $1 \otimes y^{\otimes p}$ in the zeroth cohomology group of $C_{p}$. Let $N=1+$ $\zeta_{p}+\zeta_{p}^{2}+\cdots+\zeta_{p}^{p-1}$ be the norm element in the group ring $\mathrm{F}_{p}\left[C_{p}\right]$. If $x_{1}, \ldots, x_{p} \in H^{*} X$ are homogeneous elements, which are not all equal, then the invariant $N x_{1} \otimes \cdots \otimes x_{p}$ also defines an element $1 \otimes N x_{1} \otimes \cdots \otimes x_{p}$ in the zeroth cohomology group of $C_{p}$.

THEOREM 7.8. The following formula holds where $\delta_{i, j}$ denotes the Kronecker delta: $f_{Y}^{*}\left(1 \otimes y^{\otimes p}\right)=1 \otimes y^{p}+v \otimes y^{p-1} d y+\delta_{p, 3} v \otimes \beta \lambda y$.

Proof. We write $Y_{0}$ for the space $Y$ with trivial T-action. We first prove the theorem in the special case $Y=Y_{0}$. Here the differential is zero. There is a factorization

$$
f_{Y_{0}}: \mathrm{T} / C_{p} \times Y_{0} \stackrel{i \times 1}{\longrightarrow} E \mathrm{~T} / C_{p} \times Y_{0} \stackrel{\times \Delta}{\longrightarrow} E \mathrm{~T} \times{ }_{C_{p}} Y_{0}^{p} .
$$

By this and the formula for the Steenrod diagonal, [12] p. 119 \& Errata, the result follows.

Next we prove the following formula for a general T-space:

$$
f_{Y}^{*}\left(1 \otimes N x_{1} \otimes \cdots \otimes x_{p}\right)=v \otimes d\left(x_{1} \ldots x_{p}\right) .
$$

There is a commutative diagram as follows:

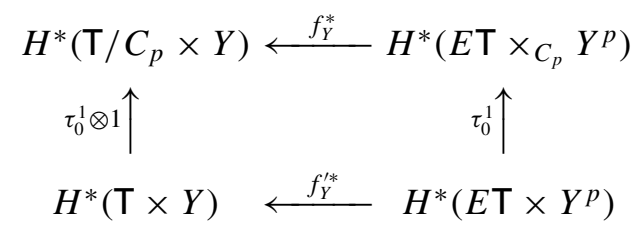


The lower horizontal map is given by

$$
f_{Y}^{\prime *}\left(1 \otimes x_{1} \otimes \cdots \otimes x_{p}\right)=\prod_{i=1}^{p}\left(1 \otimes x_{i}+v \otimes d x_{i}\right)
$$

as seen by the factorization

$$
\begin{aligned}
f_{Y}^{\prime}: \mathrm{T} \times Y \stackrel{\Delta_{2}}{\longrightarrow}(\mathrm{T} \times Y)^{2} \stackrel{p r_{1} \times \Delta_{p}}{\longrightarrow} \mathrm{T} \times(\mathrm{T} \times Y)^{p} \\
\stackrel{i \times \eta^{p}}{\longrightarrow} E \mathrm{~T} \times Y^{p} \stackrel{1 \times 1 \times \zeta_{p} \times \cdots \times \zeta_{p}^{p-1}}{\longrightarrow} E \mathrm{~T} \times Y^{p} .
\end{aligned}
$$

The norm class is hit by the transfer and by finding the coefficient to $v$ in the above formula (32) follows.

Finally we prove the Theorem for a general T-space $Y$. Because of the degrees $f_{\mathrm{T}}^{*}\left(1 \otimes v^{\otimes p}\right)=0$. The two projection maps $p r_{1}: \mathrm{T} \times Y_{0} \rightarrow \mathrm{T}$ and $p r_{2}: \mathrm{T} \times Y_{0} \rightarrow Y_{0}$ are T-equivariant. Thus we can use naturality together with the case $Y=Y_{0}$ and the above equation to find the equations below

$$
\begin{aligned}
& f_{\mathbf{T} \times Y_{0}}^{*}\left(1 \otimes(1 \otimes y)^{\otimes p}\right)=1 \otimes 1 \otimes y^{p}+\delta_{p, 3} v \otimes 1 \otimes \beta \lambda y, \\
& f_{\mathbf{T}^{\prime} \times Y_{0}}^{*}\left(1 \otimes(v \otimes 1)^{\otimes p}\right)=f_{\mathbf{T}^{\prime} \times Y_{0}}^{*}\left(1 \otimes(v \otimes d y)^{\otimes p}\right)=0 .
\end{aligned}
$$

The action map $\eta: \mathrm{T} \times Y_{0} \rightarrow Y$ is also an T-equivariant map, hence by naturality we have a commutative diagram

$$
\begin{array}{ccc}
\mathrm{T} / C_{p} \times\left(\mathrm{T} \times Y_{0}\right) \stackrel{f_{\mathrm{T} \times Y_{0}}}{\longrightarrow} E \mathrm{~T} \times \times_{C_{p}}\left(\mathrm{~T} \times Y_{0}\right)^{p} \\
1 \times \eta \downarrow \\
\mathrm{T} / C_{p} \times Y \stackrel{1 \times \eta^{p} \downarrow}{\longrightarrow} \stackrel{f_{Y}}{ } \quad E \mathrm{~T} \times \times_{C_{p}} Y^{p}
\end{array}
$$

We compute the pull back of the class $1 \otimes y^{\otimes p}$ to the cohomology of the upper left corner. First we find

$$
\begin{aligned}
\left(1 \times \eta^{p}\right)^{*}\left(1 \otimes y^{\otimes p}\right)= & 1 \otimes(1 \otimes y+v \otimes d y)^{\otimes p} \\
=1 \otimes(1 \otimes y)^{\otimes p}+1 \otimes(v \otimes d y)^{\otimes p} & \\
& \quad+\sum_{i=1}^{p-1} 1 \otimes N(1 \otimes y)^{\otimes i} \otimes(v \otimes d y)^{\otimes(p-i)} .
\end{aligned}
$$

By (32) we can compute $f_{\mathbf{T} \times Y_{0}}^{*}$ applied to the norm element terms. Only the 
$i=p-1$ term contributes.

$$
\begin{aligned}
f_{\mathrm{T} \times Y_{0}}^{*}\left(1 \otimes N(1 \otimes y)^{\otimes(p-1)} \otimes(v \otimes d y)\right)= & v \otimes d_{\top \times Y_{0}}\left(v \otimes y^{p-1} d y\right) \\
= & v \otimes\left(d_{\top}(v) \otimes y^{p-1} d y\right. \\
& \left.+v \otimes d_{Y_{0}}\left(y^{p-1} d y\right)\right) \\
= & v \otimes 1 \otimes y^{p-1} d y
\end{aligned}
$$

Altogether we have

$$
\begin{aligned}
\left(1 \otimes \eta^{*}\right) \circ f_{Y}^{*}\left(1 \otimes y^{\otimes p}\right) & =f_{\mathbf{T}^{*} Y_{0}}^{*} \circ\left(1 \times \eta^{p}\right)^{*}\left(1 \otimes y^{\otimes p}\right) \\
& =f_{\mathbf{T}^{*} \times Y_{0}}\left(1 \otimes(1 \otimes y)^{\otimes p}\right)+v \otimes 1 \otimes y^{p-1} d y .
\end{aligned}
$$

Let $\gamma: Y \rightarrow \mathrm{T} \times Y$ be the map given by $y \mapsto(1, y)$. We have $\gamma^{*} \circ \eta^{*}=1$. By applying $1 \otimes \gamma^{*}$ on both sides of the above equation the results follows.

\section{Construction of certain classes in string cohomology}

In this section $X$ denotes a connected space. We shall construct certain classes in string cohomology of $X$ from classes in ordinary cohomology of $X$.

Definition 8.1. Put $\zeta_{p}=\exp (2 \pi i / p)$ and define evaluation maps as follows:

$e v_{0}: \Lambda X \rightarrow X ; \quad \gamma \mapsto \gamma(1)$,

$e v_{1}: E \mathbf{T} \times_{C_{p}} \Lambda X \rightarrow E \mathbf{T} \times_{C_{p}} X^{p} ; \quad[e, \gamma] \mapsto\left[e, \gamma(1), \gamma\left(\zeta_{p}\right), \ldots, \gamma\left(\zeta_{p}^{p-1}\right)\right]$.

Definition 8.2. The classes $f(x), g(x), \delta(x) \in H^{*}\left(E \mathrm{~T} \times_{\mathrm{T}} \Lambda X\right)$ for $x \in$ $H^{*} X$ are defined by

$f(x)=\tau_{1}^{\infty} \circ e v_{1}^{*}\left(v \otimes x^{\otimes p}\right), \quad g(x)=\tau_{1}^{\infty} \circ e v_{1}^{*}\left(1 \otimes x^{\otimes p}\right), \quad \delta(x)=\tau_{0}^{\infty} \circ e v_{0}^{*}(x)$.

The class $u$ is defined by $u=p r_{1}^{*}(u)$ where $p r_{1}: E \mathrm{~T} \times_{\mathrm{T}} \Lambda X \rightarrow B \mathrm{~T}$ is the projection on the first factor.

THEOREM 8.3. Let $i_{0}: X \hookrightarrow \Lambda X$ denote the constant loop inclusion and let $i_{\infty}$ be the corresponding map of $\mathrm{T}$-homotopy orbits. There is a commutative diagram as follows

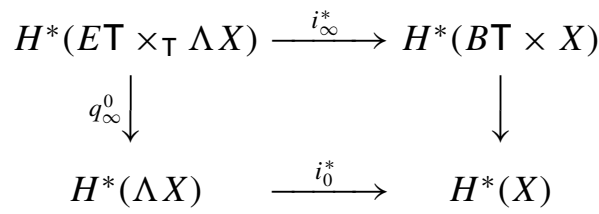


and an inclusion $\operatorname{Im}\left(q_{\infty}^{0}\right) \subseteq \operatorname{ker}\left(d: H^{*}(\Lambda X) \rightarrow H^{*}(\Lambda X)\right)$. The constructed classes are mapped as follows under $i_{\infty}^{*}$.

$$
\begin{aligned}
& i_{\infty}^{*}(f(x))=\hat{\sigma}(x) S t_{0}(x)+\sigma(x)(-1)^{m} m ! u^{m} S t_{0}(x), \\
& i_{\infty}^{*}(g(x))=\hat{\sigma}(x) S t_{1}(x)+\sigma(x)(-1)^{m} m ! u^{m-1} S t_{1}(x), \\
& i_{\infty}^{*}(\delta(x))=0 \quad \text { and } \quad i_{\infty}^{*}(u)=u \otimes 1 .
\end{aligned}
$$

Here $m=(p-1) / 2$. Under $q_{\infty}^{0}$ the images of the classes are as follows.

$$
\begin{aligned}
& q_{\infty}^{0}(f(x))=\hat{\sigma}(x) e\left(x^{p}\right), \\
& q_{\infty}^{0}(g(x))=\hat{\sigma}(x) e\left(x^{p-1} d x\right)+\sigma(x) \delta_{p, 3} e(\beta \lambda x), \\
& q_{\infty}^{0}(\delta(x))=e(d x) \quad \text { and } \quad q_{\infty}^{0}(u)=0 .
\end{aligned}
$$

Here $\delta_{p, 3}=1$ for $p=3$ and zero otherwise.

Proof. A commutative diagram of spaces gives the diagram (33) and Proposition 7.5 gives the stated inclusion.

We check that the formulas for $i_{\infty}^{*}$ are valid. Since $i_{\infty}$ sits over the identity on $B \mathrm{~T}$ we have $i_{\infty}^{*}(u)=u \otimes 1$. There is a commutative diagram as follows where $\Delta_{p}: X \rightarrow X^{p}$ is the diagonal and $i_{1}$ is the map of $C_{p}$-homotopy orbits induced by $i_{0}$.

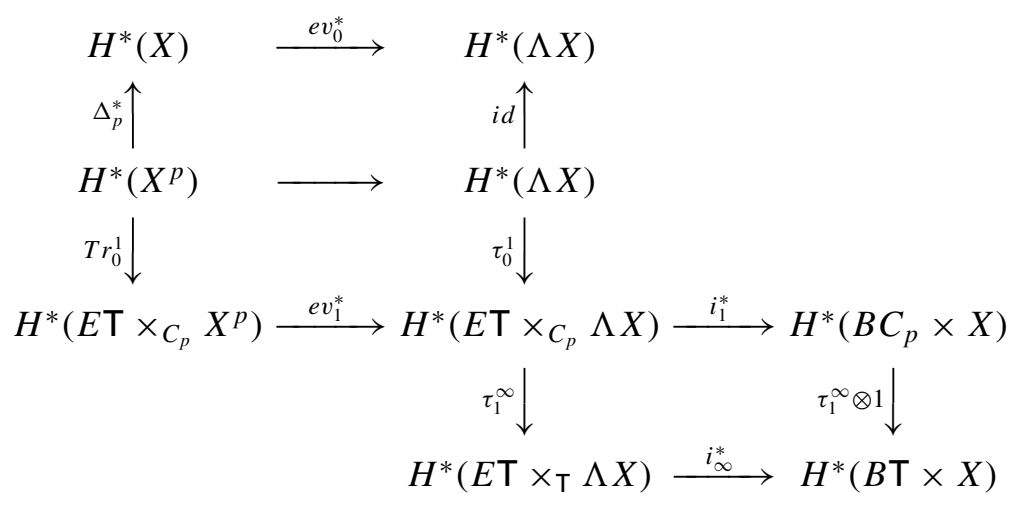

The horizontal map with no label is the induced in cohomology of the map $\gamma \mapsto\left(\gamma(1), \gamma\left(\zeta_{p}\right), \ldots, \gamma\left(\zeta_{p}^{p-1}\right)\right)$. A homotopy commutative square of spaces shows that the upper square commutes and it is obvious that the other two are commutative.

The composite $e v_{1} \circ i_{1}$ is the diagonal $\Delta_{1}$. Its induced in cohomology is the 
Steenrod diagonal $\Delta_{1}^{*}$ given by the following ([12] p. $119 \&$ Errata): $v(q) \Delta_{1}^{*}\left(1 \otimes x^{\otimes p}\right)=\sum_{i}(-1)^{i} u^{m(q-2 i)} \otimes P^{i} x+\sum_{i}(-1)^{i} v u^{m(q-2 i)-1} \otimes \beta P^{i} x$ where $q=|x|$ and $v(q)=(m !)^{q}(-1)^{m\left(q^{2}+q\right) / 2}$. From this formula and the lower part of the diagram we see that

$$
\begin{aligned}
& v(q) i_{\infty}^{*}(f(x))=\sum_{i}(-1)^{i} u^{m(q-2 i)} \otimes P^{i} x=(-1)^{[q / 2]} u^{\sigma(x) m} S t_{0}(x), \\
& v(q) i_{\infty}^{*}(g(x))=\sum_{i}(-1)^{i} u^{m(q-2 i)-1} \otimes \beta P^{i} x=(-1)^{[q / 2]} u^{\sigma(x)(m-1)} S t_{1}(x) .
\end{aligned}
$$

By [12] Lemma 6.3 one has $(m !)^{2}=(-1)^{m+1} \bmod p$ and from this one sees that $v(q)^{-1}(-1)^{[q / 2]}=1$ for $q$ even and $v(q)^{-1}(-1)^{[q / 2]}=(-1)^{m} m$ ! for $q$ odd. Hence we have verified the formulas for $i_{\infty}^{*}(f(x))$ and $i_{\infty}^{*}(g(x))$.

By the left part of the diagram we see that

$$
\delta(x)=\tau_{1}^{\infty} \circ e v_{1}^{*} \circ T r_{0}^{1}(x \otimes 1 \otimes \cdots \otimes 1) .
$$

The composite $\Delta_{1}^{*} \circ T r_{0}^{1}$ is zero by [12] Lemma 4.1 so $i_{\infty}^{*}(\delta(x))=0$.

We now check the formulas for $q_{\infty}^{0}$. It follows directly from Proposition 7.5 that $\delta(x)$ is mapped as stated and clearly $u$ is mapped to zero. For the classes $f(x)$ and $g(x)$ we proceed as follows.

Let $Y$ be a T-space and let $e$ be a point in $E \mathrm{~T}$. There is a T-equivariant map $\theta_{0}: \mathrm{T} \times Y_{0} \rightarrow E \mathbf{T} \times Y$ given by $(z, y) \mapsto(z e, z y)$ where $Y_{0}$ means $Y$ with trivial T-action. Let $\theta_{1}$ be the associated map of $C_{p}$-orbits ie. $\theta_{1}=\theta_{0} / C_{p}$. There is a commutative diagram

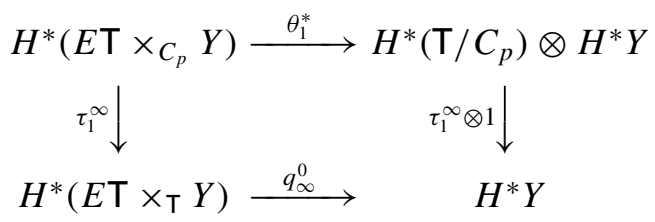

where $\tau_{1}^{\infty}: H^{*}\left(\mathrm{~T} / C_{p}\right) \rightarrow \mathrm{F}_{p}$ is given by $1 \mapsto 0$ and $v \mapsto 1$. This is proved in as similar way as [2] Proposition 4.6. When $Y=\Lambda X$ we have

$$
q_{\infty}^{0} \circ \tau_{1}^{\infty} \circ e v_{1}^{*}=\left(\tau_{1}^{\infty} \otimes 1\right) \circ \theta_{1}^{*} \circ e v_{1}^{*}=\left(\tau_{1}^{\infty} \otimes 1\right) \circ\left(e v_{1} \circ \theta_{1}\right)^{*} .
$$

Note that $e v_{1} \circ \theta_{1}$ equals the composite

$$
\mathrm{T} / C_{p} \times \Lambda X \stackrel{f_{\Lambda X}}{\longrightarrow} E \mathrm{~T} \times{ }_{C_{p}}(\Lambda X)^{p} \stackrel{1 \times e v_{0}^{p}}{\longrightarrow} E \mathrm{~T} \times_{C_{p}} X^{p}
$$


where $f_{\Lambda X}$ is the map from Definition 7.7. Thus we have

$$
q_{\infty}^{0} \circ \tau_{1}^{\infty} \circ e v_{1}^{*}=\left(\tau_{1}^{\infty} \otimes 1\right) \circ f_{\Lambda X}^{*} \circ\left(1 \times e v_{0}^{p}\right)^{*} .
$$

From this and Theorem 7.8 we get the stated results.

PROPOSITION 8.4. The following diagram is a pullback square:

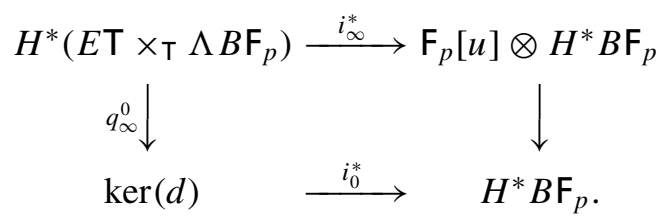

Proof. Define the action map $f_{n}: \mathbf{Z} \times \mathbf{F}_{p} \rightarrow \mathbf{F}_{p}$ by $(r,[s]) \mapsto[n r+$ $s$ ] for $n \in \mathrm{F}_{p}$. We let $B \mathrm{~F}_{p}(n)$ denote $B \mathrm{~F}_{p}$ equipped with T-action $B f_{n}$ and write $d_{(n)}$ for the corresponding action differential on $H^{*} B \mathrm{~F}_{p}(n)$. So we have $H^{*} B \mathrm{~F}_{p}(n)=\Lambda\left(v_{n}\right) \otimes \mathrm{F}_{p}\left[\beta v_{n}\right]$ where $\left|v_{n}\right|=1$.

We claim that $d_{(n)}\left(v_{n}\right)=n$ and $d_{(n)}\left(\beta v_{n}\right)=0$. Firstly, $\left(B f_{n}\right)^{*}\left(v_{n}\right)=$ $1 \otimes v_{n}+n v \otimes 1$ as one sees from $H_{1}\left(B f_{n}\right)=\pi_{1}\left(B f_{n}\right)=f_{n}$ by taking duals. Secondly, $\lambda v_{n}=v_{n}$ so $d_{(n)}\left(\beta v_{n}\right)=0$.

From [1] Lemma 7.11 we have $\Lambda B \mathrm{~F}_{p} \simeq \sqcup B \mathrm{~F}_{p}$ where the disjoint union is taken over $n \in \mathrm{F}_{p}$. Define maps as follows for $n \in \mathrm{F}_{p}$ :

$$
j_{n}: B \mathrm{~F}_{p}(n) \rightarrow \Lambda B \mathrm{~F}_{p} ; \quad x \mapsto\left(z \mapsto B f_{n}(z, x)\right) .
$$

These are T-equivariant maps. Let $\left(\Lambda B \mathrm{~F}_{p}\right)(n)$ denote the component of $\Lambda B \mathrm{~F}_{p}$ containing the image of $j_{n}$. Then the restriction $j_{n} \mid$ of $j_{n}$ to $\left(\Lambda B \mathrm{~F}_{p}\right)(n)$ is Tequivariant and a homotopy equivalence. Especially the induced in cohomo$\operatorname{logy}\left(j_{n} \mid\right)^{*}$ is an isomorphism of differential graded algebras. Thus $\left(\Lambda B \mathrm{~F}_{p}\right)(n)$ $\neq\left(\Lambda B \mathrm{~F}_{p}\right)(m)$ for $n \neq m$ since the differentials on their cohomology rings are different. Hence $\sqcup j_{n}: \sqcup B \mathrm{~F}_{p}(n) \rightarrow \Lambda B \mathrm{~F}_{p}$ is T-equivariant and a homotopy equivalence. It follows that the induced map of T-homotopy orbits $\left(\sqcup j_{n}\right)_{\infty}$ is a weak homotopy equivalence.

The diagram in the statement is via $\left(\sqcup j_{n}\right)_{\infty}$ equivalent to the following diagram:

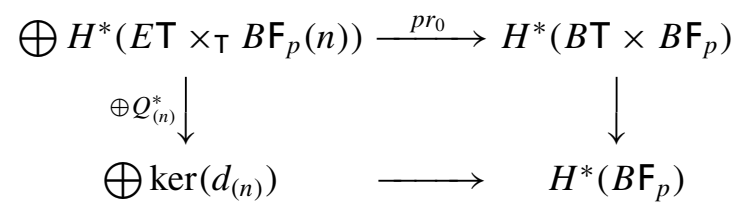

where $Q_{(n)}: E \mathrm{~T} \times B \mathrm{~F}_{p}(n) \rightarrow E \mathrm{~T} \times{ }_{\mathrm{T}} B \mathrm{~F}_{p}(n)$ denotes the quotient map and $p r_{0}$ is the projection on the direct summand with $n=0$. 
We have $H^{*}\left(E \mathrm{~T} \times_{\mathrm{T}} B \mathrm{~F}_{p}(n)\right) \cong \operatorname{ker}\left(d_{(n)}\right)$ for $n \neq 0$ since here the LeraySerre spectral sequence has $E_{3}^{i, *}=0$ for $i \geq 1$. It follows that the diagram is a pullback.

As indicated by Theorem 8.3 above it turns out that when $|x|$ is odd then both $f(x)$ and $g(x)$ can be written as a product of some power of $u$ with another class. This was not the case for $p=2$ as described in [2]. We construct new classes to get around this difficulty.

Theorem 8.5. Let $x \in H^{*} X$ be a cohomology class of odd degree. Then there exist classes $\phi(x), q(x) \in H^{*}\left(E \mathrm{~T} \times_{\mathrm{T}} \Lambda X\right)$ with $|\phi(x)|=p(|x|-1)+1$ and $|q(x)|=p(|x|-1)+2$ such that

$$
\begin{array}{ll}
i_{\infty}^{*}(\phi(x))=S t_{0}(x), & q_{\infty}^{0}(\phi(x))=\lambda x-x(d x)^{p-1}, \\
i_{\infty}^{*}(q(x))=S t_{1}(x), & q_{\infty}^{0}(q(x))=\beta \lambda x .
\end{array}
$$

Proof. It suffices to prove the theorem when $X=K\left(\mathrm{~F}_{p}, n\right)$ for odd $n \geq 1$. The general case then follows by defining $\phi(x)=\left(1 \times_{\top} \Lambda h\right)^{*} \phi\left(\iota_{n}\right)$ and $q(x)=$ $(1 \times \mathrm{T} \Lambda h)^{*} q\left(\iota_{n}\right)$ where $n=|x|$ and $h: X \rightarrow K\left(\mathrm{~F}_{p}, n\right)$ has $h^{*}\left(\iota_{n}\right)=x$. So assume that $X=K\left(\mathrm{~F}_{p}, n\right)$.

For $n=1$ we have $S t_{0}\left(\iota_{1}\right)=1 \otimes \iota_{1}$ and $S t_{1}\left(\iota_{1}\right)=1 \otimes \beta \iota_{1}$ so here the result follows from Proposition 8.4.

Assume that $n=2 r+1$ where $r \geq 1$. By Proposition 7.1, Theorem 2.9 and Theorem 4.9 the $E_{3}$-term of the Leray-Serre spectral sequence for the fibration $\Lambda X \rightarrow E \mathrm{~T} \times_{\mathrm{T}} \Lambda X \rightarrow B \mathrm{~T}$ has the following form:

$$
E_{3} \cong \operatorname{Im}(d) \oplus\left(\mathrm{F}_{p}[u] \otimes \widetilde{\Omega}(K)\right)
$$

where $K=H^{*} X$. Here $u$ has bidegree $(2,0)$ and an element $y$ in $\operatorname{Im}(d)$ or $\widetilde{\Omega}(K)$ has bidegree $(0,|y|)$. Define $s: B \mathrm{~T} \rightarrow E \mathrm{~T} \times_{\mathrm{T}} \Lambda X$ such that $p r_{1} \circ s=i d$ by choosing a constant loop. By $s^{*}$ we see that the horizontal line $(*, 0)$ survives to $E_{\infty}$.

Up to dimension $2 r p+2 p-1$ the only horizontal lines $(*, m), m \geq 0$ which are non trivial for $*>0$ are $(*, 0),(*, 2 r p+1),(*, 2 r p+2)$ and $(*, 2 r p+2 p-1)$ corresponding to powers of $u$ times the classes $1, \phi\left(\iota_{n}\right)$, $q\left(\iota_{n}\right)$ and $q\left(\beta \iota_{n}\right)$ in $\widetilde{\Omega}(K)$ respectively. Hence we can define $\phi\left(\iota_{n}\right), q\left(\iota_{n}\right)$ in $H^{*}(E \mathrm{~T} \times \mathrm{T} \Lambda X)$ by

$$
q_{0}^{\infty}\left(\phi\left(\iota_{n}\right)\right)=\lambda \iota_{n}-\iota_{n}\left(d \iota_{n}\right)^{p-1}, \quad q_{0}^{\infty}\left(q\left(\iota_{n}\right)\right)=\beta \lambda \iota_{n} \quad \text { and } \quad s^{*}\left(q\left(\iota_{n}\right)\right)=0 .
$$

Since $\left|f\left(\iota_{n}\right)\right|=2 r p+p$ and $\left|g\left(\iota_{n}\right)\right|=2 r p+p-1$ we see that $f\left(\iota_{n}\right)=$ $C_{1} u^{m} \phi\left(\iota_{n}\right)$ and $g\left(\iota_{n}\right)=C_{2} u^{m-1} q\left(\iota_{n}\right)$ where $C_{1}, C_{2} \in \mathrm{F}_{p}$ and $m=(p-1) / 2$ 
as before. By Theorem 8.3 we conclude that

$$
\begin{aligned}
C_{1} u^{m} i_{\infty}^{*}\left(\phi\left(\iota_{n}\right)\right) & =(-1)^{m} m ! u^{m} S t_{0}\left(\iota_{n}\right), \\
C_{2} u^{m-1} i_{\infty}^{*}\left(q\left(\iota_{n}\right)\right) & =(-1)^{m} m ! u^{m-1} S t_{1}\left(\iota_{n}\right)
\end{aligned}
$$

and the result follows.

Definition 8.6. For $x \in H^{*} X$ of even degree we simply define $\phi(x)=$ $f(x)$ and $q(x)=g(x)$.

\section{String cohomology and the functor $\ell$}

In this section we prove the main result of this paper:

THEOREM 9.1. Let $X$ be a connected space with $H_{*} X$ of finite type. Then there is a morphism of unstable $\mathscr{A}$-algebras

$$
\psi: \ell\left(H^{*} X\right) \rightarrow H^{*}\left(E \mathrm{~T} \times_{\mathrm{T}} \Lambda X\right)
$$

which sends $\phi(x), q(x), \delta(x)$ for $x \in H^{*} X$ and $u$ to the constructed classes with the same names. The morphism is natural in $X$. If both of the maps

$$
e: \bar{\Omega}\left(H^{*} X\right) \rightarrow H^{*}(\Lambda X), \quad \Phi: \widetilde{\Omega}\left(H^{*} X\right) \rightarrow H^{*}\left(\bar{\Omega}\left(H^{*} X\right)\right)
$$

are isomorphisms then so is $\psi$. In particular $\psi$ is an isomorphism when $H^{*} X$ is a free object in $\mathscr{K}$.

Proof. Assume that both $e$ and $\Phi$ are isomorphisms and put $K=H^{*} X$. By Theorem 4.5 we have that $\operatorname{Im}(Q)=\operatorname{ker}(d)$. From the results in Section 8 we see that $\operatorname{Im}(Q) \subseteq \operatorname{Im}\left(q_{\infty}^{0}\right)$ so $\operatorname{ker}(d) \subseteq \operatorname{Im}\left(q_{\infty}^{0}\right)$. It now follows from Proposition 7.6 that $\operatorname{ker}(d)=\operatorname{Im}\left(q_{\infty}^{0}\right)$ and that the Leray-Serre spectral sequence associated to the fibration $\Lambda X \rightarrow E \mathrm{~T} \times_{\mathrm{T}} \Lambda X \rightarrow B \mathrm{~T}$ collapses at the $E_{3}$-term:

$$
E_{\infty}=E_{3} \cong \operatorname{ker}(d) \oplus(u \otimes \widetilde{\Omega}(K)) \oplus\left(u^{2} \otimes \widetilde{\Omega}(K)\right) \oplus \cdots
$$

By Proposition 4.6 the filtration of $\ell(K)$ by powers of the ideal $(u)$ also has (34) as associated graded object. If we fix a degree the filtrations are finite and we conclude that $\ell(K)$ and $H^{*}\left(E \mathrm{~T} \times \times_{\mathrm{T}} \Lambda X\right)$ have the same dimension in each degree. Hence it suffices to show that the map $\psi$ in the statement is a well defined morphism which is surjective.

The constructed classes are algebra generators for $H^{*}\left(E \mathrm{~T} \times_{\mathrm{T}} \Lambda X\right)$ by the collapse, and the formulas for their images under $i_{\infty}^{*}$ given in Section 8 show 
that $\operatorname{Im}\left(i_{\infty}^{*}\right)=R(K)$. Hence we have a commutative diagram as follows:

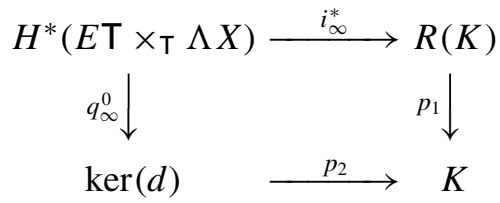

The kernel of $p_{1}$ is the ideal $(u \otimes 1)$ and $i_{\infty}^{*}(u)=u \otimes 1$. Since $u \in \operatorname{ker}\left(q_{\infty}^{0}\right)$ and $i_{\infty}^{*}$ is surjective we conclude that the restriction $i_{\infty}^{*} \mid: \operatorname{ker}\left(q_{\infty}^{0}\right) \rightarrow \operatorname{ker}\left(p_{1}\right)$ is surjective. Hence we have a surjection into the pullback.

We now restrict to the case where $H^{*} X$ is a free object in $\mathscr{K}$. Here $e$ is an isomorphism by Proposition 2.9 and $\Phi$ is an isomorphism by Theorem 4.9 and Lemma 6.1.

The above surjection into the pullback together with Theorem 6.7 gives us a surjective morphism $\psi^{\prime}: H^{*}\left(E \mathrm{~T} \times_{\mathrm{T}} \Lambda X\right) \rightarrow \ell(K)$ which is then an isomorphism. By definition it has inverse $\psi$.

By the fact that $K\left(\mathrm{~F}_{p}, n\right)$ classifies degree $n$ cohomology and naturality of the constructed classes, we can now conclude that the defining relations for $\ell(K)$ are universal for the constructed classes. Hence $\psi$ is a well defined morphism in general.

In the case where $e$ and $\Phi$ are isomorphisms, the collapse ensures that $\psi$ is surjective and hence an isomorphism.

Corollary 9.2. Let $X$ be a connected space with $H_{*} X$ of finite type. If $H^{*} X$ is a polynomial algebra on a set of even dimensional generators then $\psi$ is an isomorphism.

Proof. If $K$ is zero in odd degrees then $\bar{\Omega}(K)$ is the ordinary de Rham complex $\Omega\left(K \mid \mathrm{F}_{p}\right)$. Furthermore, $\widetilde{\Omega}(K)$ is the de Rham complex $\Omega\left(\bar{K} \mid \mathrm{F}_{p}\right)$ where $\bar{K}$ is the algebra defined by $\bar{K}^{n p}=K^{n}$ and $\bar{K}^{m}=0$ for $m \neq 0 \bmod p$. The map $\Phi$ is the Cartier map.

The Eilenberg-Moore spectral sequence for $H^{*}(\Lambda X)$ has Hochschild homology of $H^{*} X$ as its $E_{2}$-term and it collapses since the algebra generators sit in $E_{2}^{0, *}$ and $E_{2}^{-1, *}$. By the Hochschild-Konstant-Rosenberger theorem Hochschild homology is isomorphic to the de Rham complex and one concludes that $e$ is an isomorphism. The Cartier map $\Phi$ is also an isomorphism.

Remark 9.3. We have a commutative diagram which describes the ideas of our approximations:

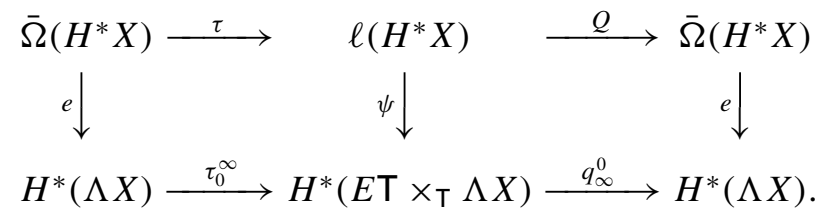




\section{Appendix: Limits and colimits in $\mathscr{F}$}

Proposition 10.1. The category $\mathscr{F}$ has all finite coproducts. The coproduct $A \otimes A^{\prime}$ of two objects $A, A^{\prime}$ in $\mathscr{F}$ is the tensor product of the underlying $\mathrm{F}_{p}$-algebras equipped with maps $\lambda * \lambda^{\prime}$ and $\beta * \beta^{\prime}$ on $A \otimes A^{\prime}$ defined by

$$
\begin{aligned}
& \lambda * \lambda^{\prime}(x \otimes y)=\lambda(x) \otimes y^{p}+x^{p} \otimes \lambda^{\prime}(y) \\
& \beta * \beta^{\prime}(x \otimes y)=\beta(x) \otimes y+(-1)^{|x|} x \otimes \beta^{\prime}(y)
\end{aligned}
$$

Proof. By direct computations one verifies that $A \otimes A^{\prime}$ is indeed an object in $\mathscr{F}$. It is then easy to see that $A \otimes A^{\prime}$ is the categorical coproduct where the canonical inclusions $i: A \rightarrow A \otimes A^{\prime}$ and $j: A^{\prime} \rightarrow A \otimes A^{\prime}$ are defined by $i(x)=x \otimes 1$ and $j(y)=1 \otimes y$.

Proposition 10.2. The category $\mathscr{F}$ is complete and cocomplete ie. all small limits and colimits exist in $\mathscr{F}$.

Proof. Similar to the proof for $p=2$ given in [11].

Proposition 10.3. The functor $\ell: \mathscr{F} \rightarrow \mathscr{A l g}$ commutes with filtered colimits. The functors $\bar{\Omega}, \widetilde{\Omega}: \mathscr{F} \rightarrow \mathscr{A l g}$ commute with all colimits.

Proof. By standard arguments $\bar{\Omega}, \widetilde{\Omega}$ and $\ell$ commute with filtered colimits. Thus it suffices to show that $\bar{\Omega}$ and $\widetilde{\Omega}$ commute with finite coproducts and coequalizers of pairs of maps [7].

For $A \in \mathscr{A} l g$ we let $D(A)$ be the free graded commutative and unital $A$ algebra on generators $d x$ for $x \in A$ of degree $|d x|=|x|-1$ modulo the ideal generated by the elements $d(x+y)-d x-d y$ and $d(x y)-d(x) y-(-1)^{|x|} x d(y)$ for $x, y \in A$. The functor $D: \mathscr{A l g} \rightarrow \mathscr{A l g}$ is left adjoint to the functor $\mathscr{A l g} \rightarrow \mathscr{A l g} ; A \mapsto \Lambda(v) \otimes A$.

The functor $D$ commutes with colimits since it is a left adjoint. In particular the canonical morphism $h: D(A) \otimes D(B) \rightarrow D(A \otimes B)$ is an isomorphism. Let $k$ denote its inverse. Using the factorization $a \otimes b=(a \otimes 1)(1 \otimes b)$ we find

$$
k(a \otimes b)=a \otimes b, \quad k(d(a \otimes b))=d(a) \otimes b+a \otimes d(b) .
$$

Now assume that $A$ and $B$ are objects in $\mathscr{F}$. We have a quotient map $D(A) \rightarrow \bar{\Omega}(A)$ inducing an isomorphism $D(A) /\left((d a)^{p}-d(\lambda a), d \beta \lambda a \mid a \in\right.$ $A) \cong \bar{\Omega}(A)$. Consider the composite map

$$
D(A \otimes B) \stackrel{k}{\longrightarrow} D(A) \otimes D(B) \longrightarrow \bar{\Omega}(A) \otimes \bar{\Omega}(B) .
$$


Elements of the form $(d(a \otimes b))^{p}+d(\lambda(a \otimes b))$ or $d(\beta \lambda(a \otimes b))$ are mapped to zero under this composite by the above formulas for $k$. So we can factor through $\bar{\Omega}(A \otimes B)$ and get an inverse to the map $\bar{\Omega}(A) \otimes \bar{\Omega}(B) \rightarrow \bar{\Omega}(A \otimes B)$.

By a similar proof one sees that $\bar{\Omega}$ commutes with coequalizers of pair of maps. Thus $\bar{\Omega}$ commutes with all colimits.

We now show that $\widetilde{\Omega}$ commutes with finite coproducts. The canonical map $h: \widetilde{\Omega}(A) \otimes \widetilde{\Omega}(B) \rightarrow \widetilde{\Omega}(A \otimes B)$ is given by $h(x \otimes y)=\widetilde{\Omega}(i)(x) \widetilde{\Omega}(j)(y)$ where $i$ and $j$ are the inclusions of $A$ and $B$ in the coproduct $A \otimes B$.

By the factorization $a \otimes b=i(a) j(b), a \in A, b \in B$ the following equations hold in $\widetilde{\Omega}(A \otimes B)$ :

$$
\begin{aligned}
\phi(a \otimes b) & =(1-\sigma(a) \sigma(b)) \phi(i(a)) \phi(j(b)) \\
q(a \otimes b) & =\hat{\sigma}(b) q(i(a)) \phi(j(b))+\hat{\sigma}(a) \phi(i(a)) q(j(b))
\end{aligned}
$$

In order to get an inverse to $h$ we define the following morphism:

$$
\begin{gathered}
k: \widetilde{\Omega}(A \otimes B) \rightarrow \widetilde{\Omega}(A) \otimes \widetilde{\Omega}(B) ; \\
\phi(a \otimes b) \mapsto(1-\sigma(a) \sigma(b)) \phi(a) \otimes \phi(b), \\
q(a \otimes b) \mapsto \hat{\sigma}(b) q(a) \otimes \phi(b)+\hat{\sigma}(a) \phi(a) q(b) .
\end{gathered}
$$

We must check that $k$ is well defined ie. that the relations (24)-(27) are respected. It suffices to consider the following special form of relation (24):

$$
\phi((x \otimes y)(z \otimes w))=(1-\sigma(x \otimes y) \sigma(z \otimes w)) \phi(x \otimes y)(z \otimes w) .
$$

We apply $k$ on the left hand side. Since $(x \otimes y)(z \otimes w)=(-1)^{\sigma(y) \sigma(z)} x z \otimes y w$ we get the element $(-1)^{\sigma(y) \sigma(z)} \phi(x) \phi(z) \otimes \phi(y) \phi(w)$ times the constant $\alpha$ below. When applying $k$ to the right hand side we get the same element times the constant $\beta$ below

$$
\begin{aligned}
& \alpha=(1-\sigma(x z) \sigma(y w))(1-\sigma(x) \sigma(z))(1-\sigma(y) \sigma(w)), \\
& \beta=(1-\sigma(x \otimes y) \sigma(z \otimes w))(1-\sigma(x) \sigma(y))(1-\sigma(z) \sigma(w)) .
\end{aligned}
$$

Thus it suffices to check that $\alpha=\beta$. If $\sigma(y)=\sigma(z)=0$ then $\alpha=\beta=$ $1-\sigma(x) \sigma(w)$. If one of $\sigma(y), \sigma(z)$ equals one and the other equals zero then $\alpha=\beta=\hat{\sigma}(w) \hat{\sigma}(x)$. If $\sigma(y)=\sigma(z)=1$ then $\alpha=\beta=0$. Hence the relation (24) is respected by $k$. A similar argument shows that the relation (25) is respected by $k$.

By additivity and symmetry it suffices to check that $k$ respects the following special form of relation (26): $\phi(\beta \lambda(a \otimes b))=q(a \otimes b)^{p}$ where $\sigma(a)=0$ and $\sigma(b)=1$. Since $\beta \lambda(a \otimes b)=a^{p} \otimes \beta \lambda b$ we see that $k$ applied to the left hand 
side equals $\phi\left(a^{p}\right) \otimes \phi(\beta \lambda b)=\phi(a)^{p} \otimes q(b)^{p}$. Since $k$ applied to the right hand side equals $(\phi(a) \otimes q(b))^{p}=\phi(a)^{p} \otimes q(b)^{p}$ the relation is respected.

By additivity and symmetry it suffices to check that $k$ respects the following special form of relation (27): $q(\beta \lambda(a \otimes b))=0$ where $\sigma(a)=0$ and $\sigma(b)=1$. We find $k(q(\beta \lambda(a \otimes b)))=\phi\left(a^{p}\right) \otimes q(\beta \lambda b)=0$ so the relation is respected. We have shown that $k$ is well defined. We have $h \circ k=i d$ and also $k \circ h=i d$ as one sees by evaluating on algebra generators. Hence $k$ is an isomorphism and $\widetilde{\Omega}$ commutes with finite products.

Finally we verify that $\widetilde{\Omega}$ commutes with coequalizers of pairs of maps. For $f, g: A \rightrightarrows B$ in $\mathscr{F}$ we have $\operatorname{coeq}(f, g)=B /(f(a)-g(a) \mid a \in A)$ and

$$
\operatorname{coeq}(\widetilde{\Omega}(f), \widetilde{\Omega}(g))=\widetilde{\Omega}(B) /(\widetilde{\Omega}(f)(x)-\widetilde{\Omega}(g)(x) \mid x \in \widetilde{\Omega}(A)) .
$$

The canonical morphism $h: \operatorname{coeq}(\widetilde{\Omega}(f), \widetilde{\Omega}(g)) \rightarrow \widetilde{\Omega}(\operatorname{coeq}(f, g))$ is given by $h[\phi(b)]=\phi([b])$ and $h[q(b)]=q([b])$. We check that there is a well defined map $k$ in the opposite direction with $k(\phi([b]))=[\phi(b)]$ and $k(q([b]))=$ $[q(b)]$.

It suffices to verify that if $y$ is an element in the ideal $(f(a)-g(a) \mid a \in A)$ then $\phi(y)$ and $q(y)$ lies in the ideal $(\widetilde{\Omega}(f)(x)-\widetilde{\Omega}(g)(x) \mid x \in \widetilde{\Omega}(A))$. Writing $x=(f(a)-g(a)) z$ for some $a \in A$ and $z \in B$ this follows directly by the relations (24) and (25). By definition $k$ is the inverse to $h$.

\section{REFERENCES}

1. Bökstedt, M., Hsiang, W. C., Madsen, I., The cyclotomic trace and algebraic K-theory of spaces, Invent. Math. 111 (1993), no. 3, 465-539.

2. Bökstedt, M., and Ottosen, I., Homotopy orbits of free loop spaces, Fund. Math. 162 (1999), 251-275.

3. Cartier, P., Une nouvelle opération sur les formes différentielles, C. R. Acad. Sci. Paris 244 (1957), 426-428.

4. Chas, M., and Sullivan, D., String Topology, Preprint 1999, available via internet from http://arxiv.org/, math. GT/9911159.

5. Lannes, J., Sur les espaces fonctionnels dont la source est le classifiant d'un p-groupe abélien élémentaire, Inst. Hautes Études Sci. Publ. Math. 75 (1992), 135-244.

6. Li, H. H., and Singer, W. M., Resolutions of Modules Over the Steenrod Algebra and Classical Theory of Invariants, Math. Z. 181 (1982), 269-286.

7. Mac Lane, S., Categories for the working mathematician, Graduate Texts in Mathematics 5, 1971.

8. Madsen, I.,Algebraic K-theory and traces, Current developments in mathematics, 1995 (Cambridge, MA), 191-321, Internat. Press, Cambridge, MA, 1994.

9. May, J. P., A general algebraic approach to Steenrod operations, The Steenrod Algebra and its Applications, 1970, 153-231, Lecture Notes in Math. 168.

10. Nishida, G., Cohomology Operations in Iterated Loop Spaces, Proc. Japan Acad. 44 (1968), 104-109.

11. Ottosen, I., Equivariant evaluations on free loop spaces, Math. Z. 239 (2002), 611-643. 
12. Steenrod, N. E., Cohomology Operations, Ann. of Math. Stud. 50, 1962.

13. Wilkerson, C., Classifying spaces, Steenrod operations, and algebraic closure, Topology 16 (1977), 227-237.

\author{
DEPARTMENT OF MATHEMATICAL SCIENCES \\ UNIVERSITY OF AARHUS \\ BUILDING 530 \\ DK-8000 ÅRHUS C \\ DENMARK \\ E-mail: ottosen@imf.au.dk
}

